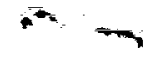 \\ NASÁ Contractor Report 181963 \\ ICASE Report No. 89-82
}

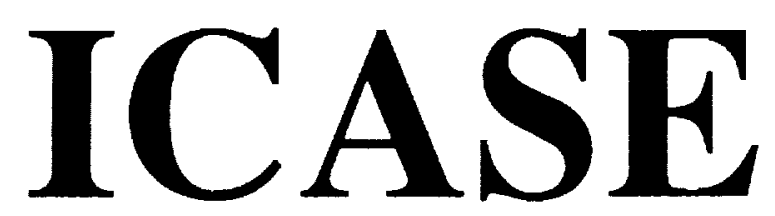

ON STRONGLY NONLINEAR VORTEX/WAVE INTERACTIONS IN BOUNDARY-LAYER TRANSITION

\author{
P. Hall \\ F. T. Smith
}

Contract No. NAS1-18605

December 1989

Institute for Computer Applications in Science and Engineering

NASA Langley Research Center

Hampton, Virginia 23665-5225

Operated by the Universities Space Research Association

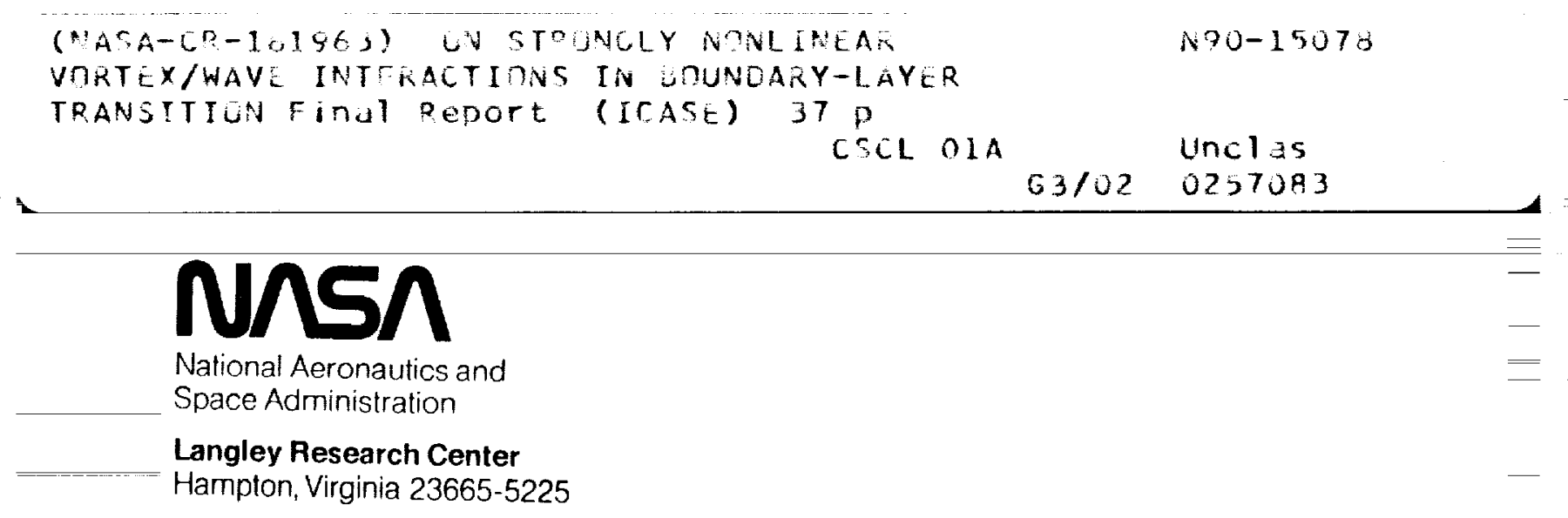

(NASA-CR-1,196,) UV STOJNCLY NONLINEAR

VORTEX/WAYE INTERACTIONS IN UOUNOARY-LAYER

CSCL OIA

$63 / 02 \quad 0257083$ 


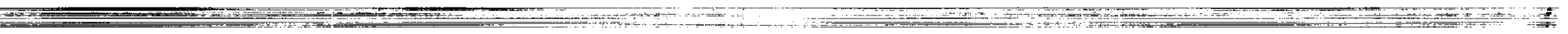

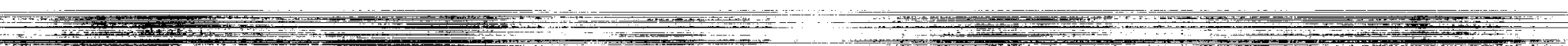

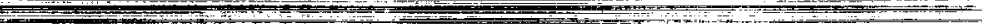

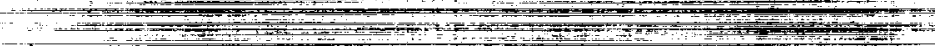

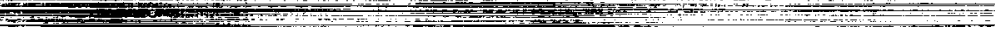

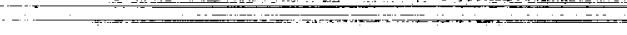




\title{
ON STRONGLY NONLINEAR VORTEX/WAVE INTERACTIONS IN BOUNDARY-LAYER TRANSITION ${ }^{1}$
}

\author{
P. Hall \\ Mathematics Department, The University \\ Exeter, EX4 4QE, UK \\ and \\ F. T. Smith \\ Mathematics Department, University College \\ Gower Street, London WC1E 6BT, UK
}

\begin{abstract}
The interactions between longitudinal vortices and accompanying waves considered here are strongly nonlinear, in the sense that the mean-flow profile throughout the boundary layer is completely altered from its original undisturbed state. Nonlinear interactions between vortex flow and Tollmien-Schlichting waves are addressed first, and some analytical and computational properties are described. These include the possibility in the spatialdevelopment case of a finite-distance break-up, inducing a singularity in the displacement thickness. Second, vortex/Rayleigh-wave nonlinear interactions are considered for the compressible boundary-layer, along with certain special cases of interest and some possible solution properties. Both types, vortex/Tollmien-Schlichting and vortex/Rayleigh, are shortscale/long-scale interactions and they have potential applications to many flows at high Reynolds numbers. Their strongly nonlinear nature is believed to make them very relevant to fully fledged transition to turbulence.
\end{abstract}

\footnotetext{
${ }^{1}$ Research was supported in part by the National Aeronautics and Space Administration under NASA Contract No. NAS1-18605 while the authors were in residence at the Institute for Computer Applications in Science and Engineering (ICASE), NASA Langley Research Center, Hampton, VA 23665.
} 


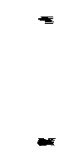

$=$

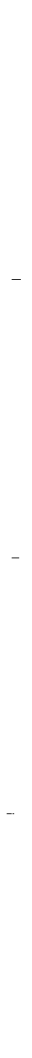




\section{INTRODUCTION}

The majority of any transition process from laminar to turbulent flow poses a considerable theoretical challenge since fully fledged transition is a strongly nonlinear process, i.e., it completely alters the mean-flow profile from its original laminar state. Much interest has therefore arisen recently in nonlinear three-dimensional (3D) interactions between longitudinal vortices and accompanying nonlinear waves, because of the strongly nonlinear nature of vortex/wave interactions and hence their likelihood of increased relevance to fully fledged transition, e.g., as observed experimentally by Klebanoff et al. (1962), Hama and Nutant (1963), Nishioka et al. (1981), Nishioka and Asai (1984, 1985), Kachanov and Levchenko (1984), Williams et al. (1984, 1987), Thomas (1987) and simulated computationally by Wray and Hussaini (1984), Kleiser and Schumann (1984), Gilbert and Kleiser (1986, 1988), Zang and Hussaini (1986, 1987), Fasel et al. (1987), Spalart and Yang (1987), Laurien and Kleiser (1989), Zang and Krist (1989). In this work vortex/wave interactions are considered theoretically in the context of boundary-layer transition.

There appear to be three main theories developed so far, of a truly nonlinear nature, in the case of flat-surface boundary layers: first, for nonlinear Tollmien-Schlichting (TS) interactions via triple-deck-like theory (e.g., Smith (1979, 1985, 1988, 1989), Hall and Smith (1984)); second, for nonlinear Euler interactions (Smith and Burggraf (1985), Smith and Stewart (1987), Smith, Doorly, and Rothmayer (1989)); and, third, for nonlinear vortex/wave interactions (Hall and Smith (1987, 1988, 1989), Bennett, Hall, and Smith (1988), Smith and Walton $(1988,1989)$, Bassom and Hall (1989)). The third, which is our concern here, can be regarded as stemming from the first, as Smith and Walton $(1988,1989)$ show. Alternatively, in the presence of curvature, longitudinal vortices are able to exist without any forcing from a wave system (Hall (1982), Hall and Lakin (1988), Hall and Seddougui (1989)). Here it is found that strongly nonlinear vortex flows completely restructure the boundary layer in a manner which renders it neutrally stable to travelling waves; the latter result is closely related to the ideas of Malkus (1950) who proposed a 'marginal theory of turbulence.' All the above theories, for high Reynolds numbers, are known to be inter-connected in that triple-deck interactions can lead on to Euler-stage flow (see Smith and Burggraf) and to vortex/wave interactions (see Hall and Smith (1987) and Smith and Walton (1988, 1989)), and further likely connections are found to arise in this study. All are particularly attractive because they can produce substantial alterations of the mean-flow profile or shear in the boundary layer, in contrast with linear instability theory and weakly nonlinear theory (e.g., Stuart-Watson-like, Benney-Lin, resonant triads, and associated near-neutral analyses) where some interesting low-amplitude phenomena can be predicted but nevertheless the mean-flow quantities are little changed. Again, the truly nonlinear interactions above are based on rational arguments 
as distinct from the interesting but ad hoc approaches of certain other theories, while the largeness of the Reynolds number taken throughout seems not unlikely to be appropriate to the experimental range of greatest concern. The question of which strongly nonlinear interaction applies in any particular experimental configuration depends on the amplitudes and spectra of the input, at an initial time and/or position. In particular, the present, vortex/wave, interactions apply for waves of small amplitude which nevertheless are able to alter the mean-flow quantities because of the relatively short wavelength, compared with the relatively large development length of the mean flow, thus inducing full nonlinearity.

The aim in this work is to describe distinct cases of vortex/wave interaction in boundarylayer flows, as well as some interesting and useful sub-cases, of which there are many. We summarize the derivations from underlying flow structures, and the governing equations, for a number of vortex/wave interactions, to focus attention on the wide range of possibilities and applications, and some solution properties are also given in the form of linear, secondary instability, weakly nonlinear, fully nonlinear similarity-type, and nonlinear breakdown phenomena, along with computational studies, as a start. A main task here however is felt to be to put the typical controlling equations of vortex/wave interaction on record, as they are believed to be of much significance and of broad application (see below).

The scales and amplitudes of the vortex flows tend to be relatively simple on the whole and can be inferred from the early Taylor-vortex calculations by say Davey (1962), whereas the waves' scales and amplitudes are usually more involved. The origins of these scales and of the corresponding flow structures are to be found in the flow properties described by Hall and Smith (1984, 1987, 1988, 1989), Bennett, Hall, and Smith (1988), Smith and Walton $(1988,1989)$, for lower-amplitude interactions. The two major kinds of nonlinear vortex/wave interaction that emerge, and are addressed below, concern what are effectively TS waves and Rayleigh waves.

Vortex/TS-wave interactions are discussed in Section 2 (see also Figure 1), where the scales, flow structure and controlling equations are presented, and in Section 3 where certain solution properties are considered. This is mainly for the incompressible boundary layer although the corresponding compressible version follows the same pattern, cf. Smith (1987), Blackaby (1990), Smith and Walton (1988, 1989). Vortex/Rayleigh-wave interactions (again see Figure 1) are then described in Sections 4 and 5 for compressible boundary layers, given the earlier groundwork, and given the susceptibility of such boundary layers to Rayleigh waves, with the incompressible and other limiting regimes then being obtainable as special cases (see Section 5 and Appendix B). A number of interesting nonlinear flow properties seem to be suggested. These include the possibilities that the vortex/TS interaction can provide a lead into vortex/Rayleigh interaction, even in the incompressible regime, that ei- 
ther interaction can produce eventually an Euler-stage flow, within a finite distance in the spatial problem or within a finite time for the temporal case, that many waves can be activated together, and that successive shorter-scale vortex-wave interactions can be provoked, producing a cascade of scales. In addition, as the whole boundary layer is changed substantially in any of these nonlinear interactions, there is potential relevance to by-pass transition throughout as well as to more gradual transitions triggered initially by linear disturbances. Further comments are presented in Section 6.

The nonlinear vortex/wave interactions studied here also hold for many other flows in principle and have numerous applications. Examples are channel flows (see also Bennett et al. (1988)), pipe flows (see also Walton (1990)), wakes, plane Couette flow, water motions, e.g., Langmuir circulations, free shear layers, separations, flow over surface roughnesses, vortex breakdown, and possibly other rotating fluid flows. The vortex/wave interaction seems to apply in fact to any flow that admits relatively short-scale waves. Among the interactions, those of Section 4 for nonlinear Rayleigh-like waves would appear to have the broadest application.

In the following, the large Reynolds number Re is the global one, based on the airfoil chord and free-stream speed in the aerodynamic context, as are the corresponding nondimensional coordinates $x, y, z$ and velocities $u, v, w$, streamwise, normal and spanwise respectively for flat-surface flow, and the time $t$. Similarly, the nondimensional density, viscosity, temperature and pressure are $\rho, \mu, T, p$ (with freestream value $p_{\infty}$ ), while $M_{\infty}, \sigma, C$ denote in turn the free-stream Mach number, the Prandtl number, and the constant in the Chapman viscosity law which is assumed for definiteness. The characteristic boundary-layer thickness is then $0\left(\operatorname{Re}^{-\frac{1}{2}}\right)$ in terms of $y\left[=\mathrm{Re}^{-\frac{1}{2}} \bar{y}\right]$, and the standard TS scalings, i.e., 3D triple-deck with $y$ for instance scaled as $\frac{3}{8}, \frac{1}{2}, \frac{5}{8}$ powers of $\mathrm{Re}^{-1}$ and $x, z$ as $\frac{3}{8}$ powers, apply in the setting of Section 2, whereas standard Rayleigh scales apply for Section 4. Throughout, the motions being considered are 3D and unsteady.

\section{NONLINEAR VORTEX/TOLLMIEN-SCHLICHTING INTERACTIONS}

The vortex/TS nonlinear interactions that we address first here are larger-scale ones, in which the mean-flow profile of the entire $0\left(\operatorname{Re}^{-\frac{1}{2}}\right)$ boundary layer is altered from its original laminar form. We start with the wide vortex. The scales involved may be derived from an order-of-magnitude reasoning as follows. The small TS waves of typical pressure amplitude $\bar{\Pi}$ say, to be determined, have the triple-deck structure, so that their streamwise and spanwise velocity perturbations are of order $\mathrm{Re}^{-\frac{1}{6}} h$ within the lower deck near the surface, where $\bar{\Pi}=\operatorname{Re}^{-\frac{1}{h}} h$ and $h$ is assumed to be small. The powers of the Reynolds number present here are those characteristic of the triple-deck, and hence of $2 \mathrm{D}$ and 3D TS waves, as set out in 
numerous previous works on TS waves alone. In particular the $z$ scale is comparable with $\mathrm{Re}^{-\frac{3}{8}}$. Forcing of the near-surface vortex flow then occurs at the amplitude-squared level, including a vortex spanwise velocity of order $\mathrm{Re}^{-\frac{1}{8}} h^{2}$. This velocity grows logarithmically at the edge of the lower-deck sublayer, as described by Hall and Smith (1988), and so it is little different in the main part of the boundary layer, the main deck. There the typical vortex dynamics is controlled by the convective-viscous balance acting on the $0(1)$ scale in $x$ and hence, since the $z$-scale is of order $\operatorname{Re}^{-\frac{3}{8}}$, the representative $w$ in the vortex has size $0\left(\operatorname{Re}^{-\frac{3}{8}}\right)$, given that the streamwise velocity is of order unity. So nonlinear interaction arises when this size of spanwise velocity is comparable with that forced by the presence of the TS waves, i.e., with the order $\operatorname{Re}^{-\frac{1}{8}} h^{2}$, from above. The critical size is therefore $h \sim \operatorname{Re}^{-\frac{1}{8}}$, corresponding to the pressure amplitude $\bar{\Pi}=R e^{-\frac{s}{3}}$ and confirming the relative smallness of the TS waves, which contrasts with their substantial impact on the main boundary-layer motion. The same estimate of size results from direct extensions of our previous work on related vortex-wave interactions (see references). The back-effect, of the induced longitudinal vortex motion on the waves themselves, is felt through the streamwise skin friction which helps to control the waves' response within the sublayer.

The expansions of the flow solutions in the lower, main, and upper decks may then be set down, accounting for the extra logarithmic terms necessary in view of the logarithmic sublayer behavior of the vortex spanwise velocity mentioned above. The vortex velocity has the form

$$
[u, v, w]=0\left[1, \operatorname{Re}^{-\frac{1}{2}}, \operatorname{Re}^{-\frac{3}{8}}\right]
$$

across the majority of the boundary layer, while the wave velocity and pressure are

$$
\begin{aligned}
{[u, v, w, p]=} & 0\left[\operatorname{Re}^{-\frac{3}{8}} \hat{\mathcal{L}}\right], \quad \hat{\mathcal{L}}=\left(\frac{1}{8} \ln \operatorname{Re}\right)^{-\frac{1}{2}}, \\
& 0\left[\operatorname{Re}^{-\frac{1}{4}}, \operatorname{Re}^{-\frac{3}{8}}, \operatorname{Re}^{-\frac{3}{8}}, \operatorname{Re}^{-\frac{3}{8}}\right] \hat{\mathcal{L}} \\
& 0\left[\operatorname{Re}^{-\frac{1}{4}}, \operatorname{Re}^{-\frac{1}{2}}, \operatorname{Re}^{-\frac{1}{4}}, \operatorname{Re}^{-\frac{3}{8}}\right] \hat{\mathcal{L}}
\end{aligned}
$$

in the upper, main, and lower decks respectively. See Figure 1. The resulting governing equations for the vortex/wave interaction in this context are therefore:

$$
\begin{gathered}
\text { (vortex }) \quad \bar{u}_{x}+\bar{v}_{\bar{y}}+\bar{w}_{Z}=0 \\
\bar{u}_{t}+\bar{u} \bar{u}_{x}+\bar{v} \bar{u}_{\bar{y}}+\bar{w} \bar{u}_{Z}=-\bar{p}^{\prime}(x)+\bar{u}_{\bar{y} \bar{y}} \\
\bar{w}_{t}+\bar{u} \bar{w}_{x}+\bar{v} \bar{w}_{\bar{y}}+\bar{w} \bar{w}_{Z}=0+\bar{w}_{\bar{y} \bar{y}}
\end{gathered}
$$

subject to

$$
\bar{u}=\bar{v}=0, \bar{w}=-\frac{\partial_{Z}}{\bar{\lambda}^{2}}\left(|P|^{2}+\alpha^{-2}\left|P_{Z}\right|^{2}\right) \text { at } \bar{y}=0
$$




$$
\begin{gathered}
\bar{u} \rightarrow u_{e}(x), \bar{v}_{\bar{y}} \rightarrow 0 \text { as } \bar{y} \rightarrow \infty ; \\
\text { (wave) } \quad P_{Z Z}-\mathcal{F} \frac{\bar{\lambda}_{Z}}{\bar{\lambda}} P_{Z}-\alpha^{2} P=\mathcal{G} A,
\end{gathered}
$$

with

$$
\bar{\lambda}=\bar{u}_{\bar{y}} \text { at } \bar{y}=0 .
$$

Here the scaled vortex velocity $(\bar{u}, \bar{v}, \bar{w})$ and skin friction $\bar{\lambda}$, and the wave pressure $P$ and displacement $-A$, are all unknown, as is the (real) wavenumber $\alpha(x)$ if the spatial development is under consideration. Also, $\bar{p}^{\prime}(x)=-u_{e} u_{e}^{\prime}(x)$ is the prescribed external pressure gradient, if any, and the spanwise scale has $z=\mathrm{Re}^{-\frac{3}{b}} Z$. The wave pressure-displacement $(P-A)$ law required to complete the system is given by solving

$$
\begin{gathered}
\left(\partial_{\tilde{y}}^{2}+\partial_{z}^{2}-\alpha^{2}\right) \tilde{p}=0, \\
\text { with } \tilde{p} \rightarrow 0 \text { in the farfield, } \\
\tilde{p} \rightarrow P, \tilde{p}_{\tilde{y}} \rightarrow-\alpha^{2} A, \text { as } \tilde{y} \rightarrow 0+,
\end{gathered}
$$

for the upper-deck response in the potential flow just outside the boundary layer, in the present incompressible regime; and the coefficients appearing in (2.3a) are defined by

$$
\begin{gathered}
\mathcal{F}=\frac{3}{2}+\frac{\xi_{0} A i^{\prime}\left(\xi_{0}\right)}{2 A i\left(\xi_{0}\right)}\left(1+\frac{K \xi_{0}}{A i^{\prime}\left(\xi_{0}\right)}\right), \mathcal{G}=(i \alpha \bar{\lambda})^{\frac{6}{3}} A i^{\prime}\left(\xi_{0}\right) / K, \\
\xi_{0}=-i^{\frac{1}{3}} \Omega /(\alpha \bar{\lambda})^{\frac{2}{3}}, K=\int_{\xi_{0}}^{\infty} A i(q) d q
\end{gathered}
$$

where $A i$ is the Airy function and, for the spatial case again, $\Omega$ (real) is the constant imposed frequency.

The wave-forcing of the vortex motion appears in the effective spanwise surface velocity in (2.2d), an amplitude-squared effect anticipated in the first paragraph of this section. The vortex-forcing of the wave, on the other hand, is through the skin-friction factor $\bar{\lambda}$ in the TS pressure equation (2.3a). In the pure spatial-development problem the terms $\bar{u}_{t}, \bar{w}_{t}$ in $(2.2 \mathrm{~b}, \mathrm{c})$ are to be omitted, and, in the background, multiple scaling $\partial_{x} \rightarrow \mathrm{Re}^{\frac{\mathrm{3}}{\mathrm{a}}} \partial_{X}+\partial_{x}$ operates, with the short-scale wave being dependent on $X$ through the form $P(x, Z) \exp (i \alpha X-i \Omega \tilde{t})$ in effect while the long-scale vortex flow is independent of $X$ [more precisely, $i \alpha X$ should be replaced by $\left.i \int \alpha d x \operatorname{Re}^{\frac{3}{3}}\right]$. The main alternative of pure temporal development has $\partial_{x}$ identically zero instead, in (2.2a-c), along with the multi-scaling $\partial_{t} \rightarrow \operatorname{Re}^{\frac{1}{4}} \partial_{t}+\partial_{t}$ where the fast-scale wave varies with $\tilde{t}$ as above, i.e., we have $P(t, Z) \exp (i \alpha X-i \Omega \tilde{t})$ in effect, but the slow-scale vortex evolution is independent of $\tilde{t}$, and $\alpha$ is then constant with $\Omega(t)$ to be determined.

The wide-vortex/TS interaction, then, is governed by the equations (2.2) - (2.4). 
The next two types of vortex/wave nonlinear interaction addressed are for the square vortex, for which the $y$ - and $z$-scales of the vortex are comparable, of order $\mathrm{Re}^{-\frac{1}{2}}$. These types have scales implied to some extent by Bennett and Hall (1987) and by an examination of the wide-vortex case for condensed $|z|$ scales. First, the square vortex has the scales

$$
[u, v, w]=0\left[1, \operatorname{Re}^{-\frac{1}{2}}, \operatorname{Re}^{-\frac{1}{2}}\right], p=\bar{p}(x)+0\left(\operatorname{Re}^{-1}\right),
$$

with $z=\operatorname{Re}^{-\frac{1}{2}} \bar{z}$ and $\bar{z}$ of $0(1)$, and the wave disturbance is now condensed inside the boundary layer, such that its velocity and pressure fields have

$$
\begin{aligned}
{[u, v, w, p]=} & 0\left[\operatorname{Re}^{-\frac{1}{2}}\right] \mathcal{L}_{1}, \mathcal{L}_{1}=\left(\frac{1}{6} \ln \operatorname{Re}\right)^{-\frac{1}{2}}, \\
& 0\left[\operatorname{Re}^{-\frac{1}{3}}, \operatorname{Re}^{-\frac{1}{2}}, \operatorname{Re}^{-\frac{1}{3}}, \operatorname{Re}^{-\frac{1}{2}}\right] \mathcal{L}_{1}
\end{aligned}
$$

in the midst of the boundary layer and in the $0\left(\mathrm{Re}^{-\frac{2}{5}}\right)$ sublayer, in turn. So here the vortex equations are

$$
\begin{gathered}
\bar{u}_{x}+\bar{v}_{\bar{y}}+\bar{w}_{\bar{z}}=0, \\
\bar{u}_{t}+\bar{u} \bar{u}_{x}+\bar{v} \bar{u}_{\bar{y}}+\bar{w} \bar{u}_{\bar{z}}=-\bar{p}^{\prime}(x)+\bar{u}_{\bar{y} \bar{y}}+\bar{u}_{\bar{z} \bar{z}}, \\
\bar{v}_{t}+\bar{u} \bar{v}_{x}+\bar{v} \bar{v}_{\bar{y}}+\bar{w} \bar{v}_{\bar{z}}=-p_{2 \bar{y}}+\bar{v}_{\bar{y} \bar{y}}+\bar{v}_{\bar{z} \bar{z}}, \\
\bar{w}_{t}+\bar{u} \bar{w}_{x}+\bar{v} \bar{w}_{\bar{y}}+\bar{w} \bar{w}_{\bar{z}}=-p_{2 \bar{z}}+\bar{w}_{\bar{y} \bar{y}}+\bar{w}_{\bar{z} \bar{z}} .
\end{gathered}
$$

These are the nonlinear Görtler-vortex equations in a boundary layer at zero Görtler number (Hall (1988)); in the presence of curvature a term proportional to $\bar{u}^{2}$ must be inserted in $(2.7 \mathrm{c})$. The boundary conditions appropriate to $(2.7 \mathrm{a}-\mathrm{d})$ are

$$
\begin{gathered}
\bar{u}=\bar{v}=0, \bar{w}=-\frac{\partial_{\bar{z}}}{\bar{\lambda}^{2}}\left[|P|^{2}+\alpha^{-2}\left|P_{\bar{z}}^{2}\right|\right] \text { at } \bar{y}=0, \\
\bar{u} \rightarrow u_{e}(x) \text { as } \bar{y} \rightarrow \infty
\end{gathered}
$$

while the coupled wave-pressure equation is

$$
P_{\bar{z}}-\mathcal{F} \frac{\bar{\lambda}_{\bar{z}}}{\bar{\lambda}} P_{\bar{z}}-\alpha^{2} P=0
$$

Here $\bar{\lambda}$ is the unknown skin friction as defined in (2.3b) and $\mathcal{F}$ is as in (2.5), but now the interaction relation of (2.4) is replaced by $A \rightarrow 0$ effectively. This new feature is due to the shortened streamwise and spanwise length scales associated with the wave, both of which are now $0\left(\operatorname{Re}^{-\frac{1}{2}}\right)$, thus suppressing the inviscid pressure feed-back from outside the boundary layer. The other new features of the present two-tier structure, for the square-vortex case, are the balance of normal and spanwise diffusion, in $(2.7 \mathrm{~b}-\mathrm{d})$, and the interpretation of $(2.7 \mathrm{c}, \mathrm{d})$ 
as a streamwise vorticity equation (on elimination of $p_{2}$ by cross-differentiation). It should be noted however that (2.8) has no solutions with $\alpha$ real if $\bar{\lambda}_{\bar{z}}=0$, and even with $\bar{\lambda}_{\bar{z}}=0$ the possibility exists that there are no solutions of the full interactive equations in this case.

The other type of nonlinear interaction involving a square vortex has some analogies with Bennett et al.'s (1989) for channel flow. Here the vortex exhibits scales as in (2.6a) again, but the wave form is distinct from $(2.6 \mathrm{~b}, \mathrm{c})$ in that the triple-deck structure is reinstated, such that

$$
[u, v, w, p]=0\left(\operatorname{Re}^{-\frac{3}{8}}\right) \hat{\mathcal{L}}+0\left(\operatorname{Re}^{-\frac{5}{8}}\right) \hat{\mathcal{L}}
$$

in the upper deck, and so on; in particular

$$
p=\left(\operatorname{Re}^{-\frac{3}{8}} P_{0}+\operatorname{Re}^{-\frac{5}{8}} P_{1}\right) \hat{\mathcal{L}}+\ldots
$$

with $P_{0}(x)$ uniform in $\bar{z}$ but $P_{1}$ depends on $x, \bar{z}$. The relative contributions of order $\mathrm{Re}^{-\frac{1}{\gamma}}$ in the wave form affect both the vortex and the TS response throughout. Thus the vortex equations are again (2.7a-f) except that the spanwise slip condition in (2.7e) is replaced by

$$
\bar{w}=-\frac{\partial_{\bar{z}}}{\bar{\lambda}^{2}}\left[P_{0} P_{1}^{*}+c . c .+\alpha^{-2}\left|P_{1 \bar{z}}\right|^{2}\right] \text { at } \bar{y}=0,
$$

and the wave-pressure equation becomes now

$$
P_{1 \bar{z} \bar{z}}-\mathcal{F} \frac{\overline{\lambda_{\bar{z}}}}{\bar{\lambda}} P_{1 \bar{z}}-\alpha^{2} P_{0}=\mathcal{G} A_{0}
$$

where $A_{0}=\alpha^{-1} P_{0}$. Here again the $y$ - and $z$-diffusion effects in the vortex motion are comparable, but the increase in the skin-friction variation across the span, relative to the wide-vortex case, causes the splitting of the TS wave response as in (2.9b). The solution of (2.11) for $P_{1}$ can be written in integral form as in Bennett et al. and substituted into the slip condition (2.10) in principle. Other scales and forms of splitting are also possible for this square-vortex type.

We move on now to the fourth type of nonlinear interaction to be considered, that for a small vortex. This arises as an interesting sub-case of (2.2) - (2.4)'s interaction, with the characteristic $\bar{y}$-scale reduced to $0(\Delta)$ say, near the surface, where the new parameter $\Delta \ll 1$. So we might expect that $\bar{u} \sim \Delta$, in an $0(\Delta)$ sublayer, with the $x$-scale reduced to the order $\Delta^{3}$ to preserve the convective-viscous balance in (2.2). Hence by continuity $\bar{v}$ is large, $0\left(\Delta^{-1}\right)$, and $\bar{w}$ is still larger, $O\left(\Delta^{-2}\right)$, provided the $Z$-scale remains intact. The nonlinear interplay between the vortex and the wave also stays intact if the scaled wave pressure $P$ is increased by an amount $\Delta^{-1}$, from (2.3), with $\alpha$ remaining $0(1)$. So here the vortex quantities are

$$
[u, v, w]=0\left[\Delta, \operatorname{Re}^{-\frac{1}{2}} \Delta^{-1}, \operatorname{Re}^{-\frac{3}{8}} \Delta^{-2}\right] \text { with } \bar{y} \sim \Delta, x \sim \Delta^{3},
$$


and the TS wave pressure is increased to

$$
p=0\left(\operatorname{Re}^{-\frac{3}{3}} \Delta^{-1}\right)
$$

to within a logarithmic factor. The governing equations for this case, in scaled form, are (2.2) with $\bar{p}^{\prime}(x)$ absent and with (2.2e) replaced by

$$
\bar{u}_{\bar{y}} \rightarrow \lambda_{1} \text { as } \bar{y} \rightarrow \infty,
$$

coupled with (2.3), (2.4) again. Here the constant $\lambda_{1}$ is the skin-friction factor for the undisturbed incident boundary layer. As $\Delta$ tends back towards $0(1)$ the wide-vortex case of (2.2) - (2.4) is approached, whereas for $\Delta$ reduced towards $0\left(\mathrm{Re}^{-\frac{1}{8}}\right)$ the small-scale interaction of Smith and Walton $(1988,1989)$ is recovered. A number of other small-vortex/wave nonlinear interactions can be derived in similar vein from the previous ones.

All the vortex/wave interactions described above are fully nonlinear in the sense defined in Section 1. In general each one needs a computational treatment, marching forward in $x$ from given starting conditions at $x=0$, say, in the spatial-development setting, or forward in $t$ from initial conditions at $t=0$ for the temporal setting. Note however that for either the temporal or spatial problem we cannot arbitrarily choose the input vortex velocity field since the associated Tollmien-Schlichting wave must satisfy an eigenrelation dependent on the initial shear stress. Some solution properties are presented below.

\section{NONLINEAR INTERACTION PROPERTIES}

Most of our interest here is in the spatial development for the wide vortex of (2.2) (2.4) and for its small-vortex form (2.13). The nonlinear flow properties of the vortex/wave interactions seem to depend to a large extent on the amplitude and spectra of the input disturbance upstream.

Certain special cases may be addressed first, as guidelines. Thus if the input comprises two oblique waves of relatively low amplitude and spanwise wavenumbers $\pm \beta$ say, then an analysis for $(2.13)$ for instance can be conducted similar to the analysis in Hall and Smith (1988) (but corrected for a logarithmic effect, like that in (2.1b), as described in a subsequent paper with Professor P. J. Blennerhassett). Such analysis yields interaction equations between the near-neutral waves' amplitudes and the induced vortex motion. The solutions of these weakly nonlinear equations in the Hall and Smith (1988) case show that, depending on $\beta$, either a finite-distance singularity is encountered or a far-downstream asymptote with exponential growth is attained. In the present context, the former would tend to reinstate the full vortex/wave system associated with (2.13), whereas the latter would lead on eventually to the longer-scale vortex/wave interaction of (2.2) - (2.4). Similarly, if the input 
wave is near-planar then analysis along the lines of Smith and Walton $(1988,1989)$ can be applied initially. For relatively small amplitudes, the solution properties found with weak nonlinearity present again tend to reactivate (2.2) - (2.4) or (2.13) in full. For non-small amplitudes, pronounced secondary instability to $3 \mathrm{D}$ modes is found, among other things, with the near-planar input, the 3D components growing initially in a form analogous to the exponential of an exponential of distance.

The above cases could be used to provide upstream starting conditions for the full vortex/wave-interaction systems (2.2) - (2.4), (2.13), e.g., at finite or large negative $x$ respectively. The ultimate downstream behavior on the other hand for the small-vortex case is considered in Smith and Walton $(1988,1989)$, where three main possibilities are raised. One is that the full interaction continues to downstream infinity and acquires a nonlinear similarity form (with the scaled $\bar{u}, P, \bar{y}, \bar{w}$ behaving as scaled $x^{\frac{2}{3}}, x^{-\frac{1}{3}}, x^{\frac{1}{3}}, x^{-\frac{2}{3}}$ in turn), properties of which are given in the last-named paper and by Walton (1990). In that event, the small-vortex/wave interaction acts as a precursor to the wide-vortex form. The second possibility concerns a 3D-strong-attachment singularity occurring in the vortex and wave solutions at finite $x$ downstream. This typically takes the form, with $n>\frac{1}{2}$,

$$
\bar{y} \sim\left(x_{A}-x\right)^{n} \overline{\bar{y}}, \bar{v} \sim\left(x_{A}-x\right)^{-n}, \bar{w} \sim\left(x_{A}-x\right)^{-2 n},
$$

on approach to a surface attachment line $x=x_{A}(Z)$-, say. Here $\bar{u}$ is typically $0(1)$. Near a valley plane $Z=Z_{1}$ for instance, where $\bar{w} \sim\left(Z-Z_{1}\right) \hat{w}(\overline{\bar{y}})$ vanishes, the cross-stream balances

$$
\bar{v}_{\overline{\bar{y}}}+\hat{w}=0, \bar{v} \hat{w}_{\overline{\bar{y}}}+\hat{w}^{2}=\hat{w}_{\overline{\bar{y}} \overline{\bar{y}}}
$$

dominate, yielding

$$
\bar{v}=\bar{\gamma}[\exp (-\bar{\gamma} \bar{y})-1], \hat{w}=\bar{\gamma}^{2} \exp (-\bar{\gamma} \bar{y}),
$$

where $\bar{\gamma}$ is a positive constant. Hence, the wave-pressure amplitude $P$ is proportional to $\left(x_{A}-x\right)^{-n}$ and also becomes singular at $x=x_{A}$. The third possibility in Smith and Walton is again a finite-distance singularity but of a $3 \mathrm{D}$-separation kind, taking place in the vortex motion, probably at a peak plane $Z=Z_{2}$ say. There

$$
\begin{gathered}
\bar{y} \sim\left(x_{B}-x\right)^{-N} Y, \bar{u} \sim\left(x_{B}-x\right)^{q} U, \\
\bar{v} \sim\left(x_{B}-x\right)^{-\Gamma}, \bar{w} \sim\left(x_{B}-x\right)^{q-1}\left(Z-Z_{2}\right),
\end{gathered}
$$

with $N=\Gamma+q-1$ positive and $q>1-2 \Gamma$, so that the local response is predominantly inviscid in a region which thickens in singular fashion as $x \rightarrow x_{B}-$. The solution has the form

$$
d U / d Y=A_{1} U^{m_{1}}+A_{2} U^{m_{2}}, m_{1}=1+N / q, m_{2}=m_{1}-1 / q
$$


where $A_{1}, A_{2}$ are constants. For example, if $\Gamma=\frac{1}{2}, q=1$, then $N=\frac{1}{2}$ and $U$ takes a $(\tan )^{2}$ form. The description (3.2a-e) holds for a finite range of $Y$, with $U$ becoming singular at $Y=Y_{1}$ say in such a way that a thinner region of extent $0(1)$, in terms of $y-\left(x_{B}-x\right)^{-N} Y_{1}$, is induced to smooth out the velocity profiles. In this $0(1)$ region, the velocities $\bar{u}, \bar{w}$ are $0(1)$ and their profiles are arbitrary, i.e., dependent on the flow history, apart from the matching with (3.2e) at the lower extremes and (2.13) for example in the upper extremes. In particular the effective boundary-layer displacement

$$
\delta^{*} \sim\left(x_{B}-x\right)^{-N}
$$

becomes large in this separation singularity, connected perhaps with the formation of a lambda (loop) vortex in practice.

The proposed attachment singularity (3.1) and separation one (3.2) apply equally well to the wide-vortex case in principle. The similarity form mentioned previously does not apply, however, and appears at first sight to be replaced by the far-downstream behavior

$$
\begin{gathered}
P \sim P_{0} x^{-\frac{s}{4}}+P_{1} x^{-\frac{b}{4}}+\ldots, \alpha \sim \alpha_{0} x^{-\frac{1}{4}}, \\
\bar{u} \sim 1, \bar{v} \sim x^{-\frac{1}{2}}, \bar{w} \sim x^{-1}, \lambda \sim x^{-\frac{1}{2}},
\end{gathered}
$$

suggested by (2.2) - (2.4), at least if the $Z$-scale stays fixed at $0(1)$, e.g., with $Z$-periodicity present. Here $P_{0}$ is independent of $Z$, and the splitting in (3.3a) and the rest of the solution structure bear resemblance to the square-vortex form in (2.9)-(2.11), although now $\left|\xi_{0}\right|$ is large $\left(\sim x^{\frac{1}{2}}\right)$, so that relatively high-frequency features apply. Conversely, a low-frequency input with $\Omega \ll 1$ would produce the form in (3.3), downstream, with $x$ replaced by $\Omega^{-2}$ in effect. Further investigation however suggests that there are no high-frequency solutions with $\left|\xi_{0}\right|$ large, e.g., from analysis of (2.3a) with (2.5a-d). Instead, the flow solution seems more likely to terminate with a finite-distance singularity, as in (3.1), (3.2) or with the wavepressure amplitude tending to zero in a square-root fashion, in view of $(2.2 \mathrm{~d})$, similar to a special case given in Smith and Walton $(1988,1989)$. Other possibilities for the wide-vortex and small-vortex nonlinear interactions with TS waves may exist of course, and likewise for the square-vortex forms in Section 2.

Computational studies have been made of both the wide- and the small-vortex/wave interactions of (2.2) - (2.4) and (2.13) respectively. Spectral treatments for the $Z$-variation have been applied to each case, and a finite-difference $Z$-representation has also been applied to the small-vortex case. These studies are being continued. Here we report on some preliminary but significant results obtained in our numerical investigation of the wide-vortex/wave interaction problem. This was done using a mixed finite-difference/spectral approximation 
to the vortex/wave equations (2.2) - (2.4). Thus, for example $\bar{u}$ is written as

$$
\bar{u}=\bar{u}_{0}(x, \bar{y})+\sum_{1}^{\infty} \bar{u}_{n}(x, \bar{y}) \cos n \beta Z,
$$

and the $x, \bar{y}$ dependences of $\bar{u}_{n}$ are then approximated using finite differences. All of our calculations were for the zero pressure gradient case, $\bar{p}^{\prime}=0$, but the scheme as described could be carried out for pressure-gradient-driven boundary layers.

Suppose then that $\bar{u}, \bar{v}$ and $\bar{w}$ together with $P$ and $A$, and $\alpha, \Omega$, are known at $x=\bar{x}$; we now describe a scheme which can be used to advance the solution to $x=\bar{x}+\bar{\varepsilon}$ in such a way that the wave frequency $\Omega$ is held fixed. Thus we are assuming that as the wave evolves its frequency stays constant whilst its wavelength and amplitude vary.

In order to step the solution forward, we decouple the vortex and wave equations by making $w$ at $x=\bar{x}+\bar{\varepsilon}$ satisfy the required boundary condition at $\bar{y}=0$ evaluated in terms of $P$ known at $x=\bar{x}$. The $x$ and $z$ momentum equations for the combined mean flow-vortex field can then be stepped forward using essentially the scheme used by Hall (1988) in an investigation of fully nonlinear Görtler vortices. The reader is referred to that paper for precise details of that scheme. It suffices here to say that $\bar{y}$ derivatives are approximated using central differences and the nonlinear terms involving any harmonic content of $\bar{u}, \bar{v}$ and $\bar{w}$ are iterated upon until a converged solution is found.

The above procedure is used to advance the vortex velocity field to $x=\bar{x}+\bar{\varepsilon}$ and the corresponding shear stress at that point can then be found from $\bar{u}$. The eigenrelation specified by $(2.3 \mathrm{a})$ and $(2.4 \mathrm{a}, \mathrm{b}, \mathrm{c})$ will, for fixed frequency, determine a complex value for the streamwise wavenumber $\alpha$. However, it is implicit in our analysis that the wavenumber $\alpha$ is real, so that the eigenrelation at $x=\bar{x}+\bar{\epsilon}$ in general does not have an acceptable solution. At this stage there are two simple procedures which can be used to remedy the situation. Firstly, the value of $\bar{w}(\bar{y}=0)$ at $x=\bar{x}+\bar{\varepsilon}$ can be iterated upon in order to make $\alpha$ calculated at $\bar{x}+\bar{\varepsilon}$ purely real. In effect this is most easily done by iterating upon some measure of the Tollmien-Schlichting wave amplitude at $x=\bar{x}+\bar{\epsilon}$. Alternatively, we proceed by writing $(2.3 \mathrm{a})$ in the form

$$
\left\{P_{Z Z}-\frac{1}{2} \frac{\mathcal{F} \lambda_{Z}}{\bar{\lambda}} P_{Z}-\alpha^{2} P-\frac{\mathcal{G} A}{2}\right\}^{+}=\left\{\frac{1}{2} \frac{\mathcal{F} \bar{\lambda}_{Z}}{\bar{\lambda}} P_{Z}+\frac{\mathcal{G} A}{2}\right\}^{-}
$$

where - and + denote quantities evaluated at $\bar{x}$ and $\bar{x}+\bar{\varepsilon}$ respectively. The right-hand side of (3.5) is then known, so that after expanding $P$ in a Fourier series (3.5) can be solved for $P$ at $\bar{x}+\bar{\varepsilon}$. The value of $\alpha$ used to determine $P$ is then iterated upon in order to make (say) $(P)_{i}=0$ at $Z=0$. In this procedure the Tollmien-Schlichting frequency is of course fixed and a value for $\alpha$ at $\bar{x}+\bar{\varepsilon}$ is obtained. 
The calculations which we report on have been carried out using the second of the procedures described above in order to keep $\alpha$ real. The input used to begin our calculations was found in the following way. Firstly, we assume some form for $\bar{u}$ at an initial value $x=x^{*}$ and then solve the eigenvalue problem determined by $(2.3 \mathrm{a}),(2.4 \mathrm{a}, \mathrm{b}, \mathrm{c})$ for the appropriate real values of $\alpha$ and $\Omega$. The values of $\alpha$ and $\Omega$ calculated in this way depend on $\bar{u}$ through the wall shear $\bar{\lambda}$, so that for example an almost $2 \mathrm{D}$ input Tollmien-Schlichting wave can be constructed by choosing $\bar{u}_{n}$ in (3.4) for $n \geq 1$ to be small compared with $\bar{u}_{0}$. Having solved for the Tollmien-Schlichting eigenrelation at $x=x^{*}$ the boundary condition to be satisfied by $\bar{w}$ then can be determined in terms of $P$. The initial profile for $\bar{w}$ at $x=x^{*}$ is then chosen to be consistent with this condition. In the calculations reported here $\beta=0.02, x^{*}=55$, and 8 Fourier modes were retained in the Fourier expansion of the vortex and Tollmien-Schlichting fields. The initial distribution for $\bar{u}$ was taken to be

$$
\bar{u}=u_{B}+\Delta\left\{\sum_{1}^{\infty} u_{0}^{*}(\bar{y}) \cos n \beta Z\right\}
$$

where $u_{B}$ is the Blasius profile and, $u_{0}^{*}=e^{-\bar{y}}-e^{-2 \bar{y}}$ and $\Delta$ a parameter which can be varied so as to alter the size of the incoming vortex. The spanwise velocity $\bar{w}$ was then taken to be

$$
\bar{w}=\sum_{1}^{\infty} \chi_{n} w_{0}^{*}(\bar{y}) \sin n \beta z
$$

with $w_{0}^{*}=\cos \bar{y} e^{-\bar{y}}$ and $\chi_{n}$ chosen so that $\bar{w}$ satisfies the boundary condition on $\bar{w}$ at $\bar{y}=0$ determined in terms of the Tollmien-Schlichting pressure. Clearly the above initial conditions are rather arbitrary but this is always the case with longitudinal vortex calculations unless the receptivity problem is discussed; see Hall $(1983,1989)$. Some limited experimentation with other initial conditions produced qualitatively similar results but we do not claim to have made an exhaustive investigation of the effects of the initial conditions on the vortex/wave interactions.

In order to monitor the evolution of the vortex and the wave, the following quantities were calculated as the flow was allowed to develop:

$$
e_{n}=\int_{0}^{\infty}\left(\bar{u}_{n}^{2}+\bar{w}_{n}^{2}\right) d y, \quad n=1,2, \ldots
$$

and $P_{n}$ where

$$
P=P_{0}+\sum_{1}^{\infty} P_{n} \cos n \beta Z \text {. }
$$

In Figure 2 we show results from a calculation where the initial wave is almost two-dimensional. Figures 2a,b show the development of $e_{1}, e_{2}, e_{3}, e_{4}$ and $P_{0}, P_{1}, \ldots, P_{4}$ respectively. Figure 2c shows the corresponding development of $\alpha$. The calculations were started from $\bar{x}=55$ with 
more three-dimensional as it moves downstream and that at a finite value $x$ a singularity appears to arise, beyond which the solution cannot be calculated. The apparent singularity occurred in the same place when the finite-difference resolution was increased or the spectral resolution decreased. It was not possible for us to perform the calculations with more than eight Fourier modes because of our limited computing resources. However, we believe that Figure 2 represents a significant calculation because it demonstrates the three-dimensional secondary instability, and ultimate breakdown, of an initially two-dimensional wave and the induced vorticity structure. The resolution of the calculations was not great enough to determine whether the singularity encountered numerically is related to the ones discussed earlier in this section.

In Figure 3 we show the results of a similar calculation but with smaller initial strengths for the vortex and wave fields. Here the vortex and waves decay as $x$ increases. We note that the wave appears to effectively disappear before the vortex field. This is consistent with there being a subcritical bifurcation of a three-dimensional wave from a longitudinal vortex velocity field at a finite value of $x$ (see our earlier comment concerning a square-root wave-pressure behavior).

\section{NONLINEAR VORTEX/RAYLEIGH INTERACTIONS}

Nonlinear interactions between longitudinal vortex flow and inviscid Rayleigh waves are considered here for the compressible or incompressible boundary layer, with, as a result, the boundary layer's $0(1)$ mean-flow profile again being changed completely from its original form.

The scales involved may be deduced mostly from a first-principles argument. Thus if the induced Rayleigh wave has pressure amplitude $\bar{\pi}$, its typical velocity amplitudes are also of order $\bar{\pi}$, by its inviscid nature, and the representative wavelengths are all of the short-scale size $\mathrm{Re}^{-\frac{1}{2}}$. Hence the nonlinear inertial effect provoking a mean-flow correction is of order $\bar{\pi}^{2} \mathrm{Re} \frac{\frac{1}{2}}{2}$, e.g., from $u u_{x}, u v_{x}, u w_{x}$. This is to be compared with the minimum inertial force in the typical long-scale vortex motion, namely $\mathrm{Re}^{-\frac{1}{2}}$, for a full "square" vortex of size as in (2.6a); this force is from the spanwise and normal momentum of the vortex, e.g., $u v_{x}, u w_{x}$ (and the viscous forces such as $\operatorname{Re}^{-1} w_{\bar{y}} \bar{y}$ ), rather than the strong streamwise momentum force of order unity. So the wave affects the mean flow at zeroth order if $\bar{\pi}^{2} \mathrm{Re}^{\frac{1}{2}}$ is comparable with $\mathrm{Re}^{-\frac{1}{2}}$, i.e., if the wave pressure has amplitude $\bar{\pi} \sim \operatorname{Re}^{-\frac{1}{2}}$ (smaller than in Section 2), in principle. There is a complication, however, similar to that in the vortex/TS case, namely the appearance of logarithmic behavior in the induced vortex velocities close to the (linear) critical layer(s), situated at $\bar{y}=f$ say. The logarithmic response arises because the 3D wave velocities there grow like $(\bar{y}-f)^{-1}$ (see below), so that the nonlinear inertial spanwise 
wave velocities there grow like $(\bar{y}-f)^{-1}$ (see below), so that the nonlinear inertial spanwise forcing is proportional to $(\bar{y}-f)^{-2}$, which can be balanced only by the viscous term $w_{\overline{v y}}$ of the vortex if $w$ is proportional to $\bar{l} n|\bar{y}-f|$. Hence logarithmic contributions are drawn into play again, slightly altering the interactive balances.

In consequence the flow solution in the nonlinear vortex/compressible-Rayleigh interaction (see also Figure 1) has the underlying form

$$
\begin{gathered}
{[u, v, w, p, \rho, T, \mu]=\left[\bar{u}, \operatorname{Re}^{-\frac{1}{2}} \bar{v}, \operatorname{Re}^{-\frac{1}{2}} \bar{w}, \bar{p}(x)+\operatorname{Re}^{-1} \bar{p}_{2}, \bar{\rho}, \bar{T}, \bar{\mu}\right]} \\
+\operatorname{Re}^{-\frac{1}{2}}\left[u^{(1)}, v^{(1)}, w^{(1)}, p^{(1)}, \rho^{(1)}, T^{(1)}, \mu^{(1)}\right] \mathcal{L}_{1}+\ldots
\end{gathered}
$$

where the first square brackets describe the vortex motion and the second the wave, while $\mathcal{L}_{1}=\left(\frac{1}{6} \ln R e\right)^{-\frac{1}{2}}$ is small. The expansion (4.1) applies across most of the boundary layer, with $\bar{y}, \bar{z} \sim 1$, and leads to the compressible vortex equations

$$
\begin{aligned}
& (\bar{\rho} \bar{u})_{x}+(\bar{\rho} \bar{v})_{\bar{y}}+(\bar{\rho} \bar{w})_{\bar{z}}=0, \\
& \bar{\rho}\left(\bar{u} \bar{u}_{x}+\bar{v} \bar{u}_{\bar{y}}+\bar{w} \bar{u}_{\bar{z}}\right)=-\bar{p}^{\prime}(x)+\left(\bar{\mu} \bar{u}_{\bar{y}}\right)_{\bar{y}}+\left(\bar{\mu} \bar{u}_{\bar{z}}\right)_{\bar{z}}, \\
& \bar{\rho}\left(\bar{u} \bar{v}_{\bar{x}}+\bar{v} \bar{v}_{\bar{y}}+\bar{w} \bar{v}_{\bar{z}}\right)=-p_{2 y}+\left(\bar{\mu} \bar{u}_{\bar{y}}\right)_{x}+\left(2 \bar{\mu} \bar{v}_{\bar{y}}\right)_{\bar{y}}+\left(\bar{\mu}\left(\bar{w}_{\bar{y}}+\bar{v}_{\bar{z}}\right)\right)_{\bar{z}}, \\
& \bar{\rho}\left(\bar{u} \bar{w}_{x}+\bar{v} \bar{w}_{\bar{y}}+\bar{w} \bar{w}_{z}\right)=-p_{2 \bar{z}}+\left(\bar{\mu} \bar{u}_{\bar{z}}\right)_{x}+\left(\bar{\mu}\left(\bar{w}_{\bar{v}}+\bar{v}_{\bar{z}}\right)\right)_{\bar{v}}+\left(2 \bar{\mu} \bar{w}_{\bar{z}}\right)_{\bar{z}}, \\
& \bar{\rho} \bar{T}=1, \quad \bar{\mu}=C \bar{T}, \\
& \bar{\rho}\left(\bar{u} \bar{T}_{x}+\bar{v} \bar{T}_{\bar{y}}+\bar{w} \bar{T}_{\bar{z}}\right)=\sigma^{-1}\left\{\left(\bar{\mu} \bar{T}_{\bar{y})_{\bar{y}}}+\left(\bar{\mu} \bar{T}_{\bar{z}}\right)_{\bar{z}}\right\}\right.
\end{aligned}
$$

with

$$
\begin{gathered}
\bar{u}=\bar{v}=\bar{w}=0, \quad \bar{T}=T_{w} \text { at } \bar{y}=0, \\
\bar{u} \rightarrow u_{e}(x), \bar{T} \rightarrow 1, \bar{v}_{\bar{y}} \rightarrow 0 \text { as } \bar{y} \rightarrow \infty
\end{gathered}
$$

and to the compressible Rayleigh equation for the effective wave pressure $\tilde{p}$,

$$
\tilde{p}_{\bar{y} \bar{y}}+\tilde{p}_{\bar{z} \bar{z}}-\frac{2}{\bar{M}}\left(\bar{M}_{\bar{y}} \tilde{p}_{\bar{y}}+\bar{M}_{\bar{z}} \tilde{p}_{\bar{z}}\right)-\alpha^{2}\left(1-\bar{M}^{2}\right) \tilde{p}=0
$$

with

$$
\tilde{p} \rightarrow 0 \text { (or outgoing waves) as } \bar{y} \rightarrow \infty, \tilde{p}_{\bar{y}}=0 \text { at } \bar{y}=0 .
$$

Here the wave has $p^{(1)}=\tilde{p} \exp (i \alpha X-i \Omega \tilde{t})+c . c .$, with $\alpha, \Omega(=\alpha c)$ real, the amplitude $\tilde{p}$ is independent of the faster scales $X=\operatorname{Re}^{\frac{1}{2}} x, \tilde{t}=\operatorname{Re}^{\frac{1}{2}} t$, and similarly for $u^{(1)}$, $v^{(1)}$, etc., leading to the inviscid response in (4.3). Also, $\bar{p}^{\prime}=-u_{e} u_{e}^{\prime}$ is the external pressure gradient, and $p_{2}=\bar{p}_{2} / \gamma M_{\infty}^{2}-\left(\bar{\mu}^{\prime}-\frac{2}{3} \bar{\mu}\right)\left(\bar{u}_{x}+\bar{v}_{\bar{\nu}}+\bar{w}_{\bar{z}}\right)$. The nonlinear interaction occurs through the definition of $\bar{M}$ required for (4.3),

$$
\bar{M}=(\bar{u}-c) \bar{\rho}^{\frac{1}{2}} M_{\infty}
$$


and through the boundary conditions on the vortex flow at the critical layer

$$
\bar{q}=-\frac{\gamma^{-2} \bar{\mu}^{-1} \Delta^{-\frac{3}{2}}}{M_{\infty}^{2}\left(\bar{M}^{\prime}\right)^{2} \alpha^{2}}\left\{\alpha^{2} \partial_{\bar{z}}\left(|\tilde{p}|^{2}\right)+\Delta^{-1} \partial_{\bar{z}}\left(\left|\tilde{p}_{\bar{z}}\right|^{2}\right)-4 \Delta^{-2} f_{\bar{z}} f_{\bar{z} \bar{z}}\left|\tilde{p}_{\bar{z}}\right|^{2}\right\}
$$

at

$$
\bar{y}=f(x, \bar{z}) \pm[\text { where } \bar{u}=c] .
$$

Here $\bar{q}$ is the cross-flow velocity tangential to the critical-layer curve $\bar{y}=f(x, \bar{z})$, so that

$$
\bar{q}=\Delta^{\frac{1}{2}} \bar{w}, \bar{v}=f_{\bar{z}} \bar{w}, \text { at } \bar{y}=f,
$$

and $\Delta=1+f_{\frac{2}{2}}^{2}$. The contribution (4.4a) describes the vortex-forcing of the wave, in essence, while the cross-flow slip velocity $(4.4 \mathrm{~b})$ represents the main back-effect of the wave on the vortex motion, thus producing nonlinear interaction in which both the mean flow and the wave are unknown.

The main details behind the slip condition (4.4b) are presented in Appendix A.

The nonlinear vortex/Rayleigh interaction is given by (4.2) - (4.4), for the compressible boundary layer. The pure spatial-development case is shown above, that for pure temporal development having $\bar{u} \bar{u}_{x}$ replaced by $\bar{u}_{t}$, etc., and a fixed surface temperature is assumed as an example. Some of the comments in Section 2 apply here as well, and further points about the flow structure are the following. First, the induced slip velocity in (4.4b) clearly has a connection with that for TS waves in Section 2 corresponding to $f=0$ and the highfrequency regime (see also Section 6 ). Second, the relatively thin critical layer surrounding $\bar{y}=f$ is of the linear viscous kind, because the wave pressure amplitude $\bar{\pi}$ is only $0\left(\operatorname{Re}^{-\frac{1}{2}} \mathcal{L}_{1}\right)$ (see references), and the thickness is of order $\mathrm{Re}^{-\frac{2}{3}}$. From (4.3a), (4.4a), the wave response nearby as $\bar{y} \rightarrow f \pm$ is of the form

$$
\tilde{p} \sim \tilde{p}_{0}+s \tilde{p}_{1}+s^{2} \tilde{p}_{2}+s^{3} \tilde{p}_{3}+\ldots
$$

where $s=\bar{y}-f$, in general logarithmic terms must be absent to allow both $\alpha, c$ to remain real, and

$$
\bar{M} \sim s \bar{M}_{1}+s^{2} \bar{M}_{2}+\ldots,
$$

with the coefficients above being $z$-dependent and related by

$$
\begin{gathered}
\tilde{p}_{1} \Delta=\tilde{p}_{0 \bar{z}} f_{\bar{z}}, \\
-2 \tilde{p}_{2} \Delta-f_{\bar{z} \bar{z}} \tilde{p}_{1}+\frac{2 \bar{M}_{1 \bar{z}}}{\bar{M}_{1}}\left(f_{\bar{z}} \tilde{p}_{1}-\tilde{p}_{0 \bar{z}}\right)+\tilde{p}_{0 \bar{z} \bar{z}}-\alpha^{2} \tilde{p}_{0}=0
\end{gathered}
$$




$$
\begin{aligned}
& -2 \tilde{p}_{2 \bar{z}} f_{\bar{z}}-2 \tilde{p}_{2}\left(f_{\bar{z} \bar{z}}-3 \Delta \frac{\bar{M}_{2}}{\bar{M}_{1}}+2 \frac{\bar{M}_{1 \bar{z}}}{\bar{M}_{1}} f_{\bar{z}}\right)+\tilde{p}_{1 \bar{z} \bar{z}}+\frac{2}{\bar{M}_{1}}\left(\bar{M}_{2} f_{\bar{z}}-\bar{M}_{1 \bar{z}}\right) \tilde{p}_{1 \bar{z}} \\
& +\tilde{p}_{1}\left(-\alpha^{2}+\frac{2 \bar{M}_{2 \bar{z}}}{\bar{M}_{1}} f_{\bar{z}}-\frac{\bar{M}_{2}}{\bar{M}_{1}} f_{\bar{z} \bar{z}}\right)+\left[\bar{M}_{2}\left(\tilde{p}_{0 \bar{z} \bar{z}}-\alpha^{2} \tilde{p}_{0}\right)-2 \bar{M}_{2 \bar{z}} \tilde{p}_{0 \bar{z}}\right] / \bar{M}_{1}=0 .
\end{aligned}
$$

(In principle these give three equations fixing the three terms $\tilde{p}_{0}, \tilde{p}_{1}, \tilde{p}_{2}$ ). Hence it follows that the $3 D$ wave velocities are given locally by

$$
\begin{aligned}
& \tilde{u} \sim F_{-1} s^{-1}+F_{0}+\ldots, \\
& \tilde{v} \sim G_{-1} s^{-1}+G_{0}+\ldots, \\
& \tilde{w} \sim H_{-1} s^{-1}+H_{0}+\ldots,
\end{aligned}
$$

as anticipated at the start of this section; in addition $\tilde{\rho} \sim-\bar{\rho}_{\bar{z}} \tilde{p}_{0 \bar{z}} /\left(\gamma \alpha^{2} \bar{M}_{1}^{2} \Delta s^{2}\right)$. Here the inviscid disturbance equations show that

$$
\begin{gathered}
\gamma M_{\infty}^{2} \bar{\rho}_{0} G_{-1}=-\tilde{p}_{1} / i \alpha \bar{u}_{1} \\
\gamma M_{\infty}^{2} \bar{\rho}_{0} H_{-1}=\left(f_{\bar{z}} \tilde{p}_{1}-\tilde{p}_{0 \bar{z}}\right) / i \alpha \bar{u}_{1} \\
\gamma M_{\infty}^{2} \bar{\rho}_{0} F_{-1}=-H_{-1 \bar{z}} / i \alpha
\end{gathered}
$$

consistent with (4.6), where $\bar{\rho} \sim \bar{\rho}_{0}+\bar{\rho}_{1} s+\ldots, \bar{u}-c \sim \bar{u}_{1} s+\bar{u}_{2} s^{2}+\ldots$. See also Appendix B for the incompressible regime. The singular behavior (4.7) is responsible for the generation, at the amplitude-squared level, of the logarithmic-flow effect which leads to the effective slip in (4.4b), via the $0\left(\operatorname{Re}^{-\frac{3}{3}}\right)$ critical layer (see Appendix A). Third, the 3D-wave condition in (4.6) can be shown to agree with the generalized inflexion-point condition for $2 \mathrm{D}$ or 3D simple waves on a parallel flow (and with other simpler cases). The latter waves can act as triggering mechanisms for the present nonlinear interaction. Fourth, the continuity properties of the total mean flow $\bar{u}, \bar{v}, \bar{w}$ across the critical layer are worth noting. These are that $\bar{u}, \bar{u}^{\prime}, \bar{u}^{\prime \prime}, \bar{v}, \bar{v}^{\prime}, \bar{w}$ are all continuous, with discontinuities appearing first in $\bar{u}^{\prime \prime \prime}, \bar{v}^{\prime \prime}, \bar{w}^{\prime}$ (the prime denotes the normal derivative), and requiring higher-order smoothing within the critical layer, which is addressed in Appendix A. Fifth, the wave's inertial effects on the mean flow are felt solely in the slip condition (4.4b) to leading order, the effects in the rest of the boundary layer being negligible (just!) due to the logarithmic response described earlier.

\section{SPECIAL CASES AND LIMIT SOLUTIONS}

The vortex/compressible-Rayleigh-wave nonlinear interaction set up in Section 4 poses a computational task, in general, which seems a particularly severe one in view of the unknown 
moving boundary present (in (4.4)) and the coupled partial-differential systems (4.2), (4.3). No full solutions have been obtained yet, and appropriate computational treatments are only just being considered. For that reason we turn briefly here to certain special or limiting properties in (i) - (vii) below, to help provide some possible guidelines and suggestions.

(i) The linearized version, where $\bar{w}$ and $\partial_{x}$ are identically zero in (4.2) and $\bar{u}, \bar{v}$ take on the compressible Blasius form, say, applies at small wave-amplitudes $|\tilde{p}|$ and leaves the linear compressible-Rayleigh equation (4.3) controlling instability, with $\bar{M}$ known in advance. Solution properties at various Mach numbers are given by Mack (1975, 1984) and Malik $(1982,1987)$ principally. The main result in the present context is that neutral modes exist at all Mach numbers (see, e.g., Mack's (1984) figures) and so can act as triggers for the nonlinear interaction of Section 4. See also (iv) below.

(ii) Weak nonlinearity can also be handled analytically in principle, an example being for two input oblique waves of lowish amplitudes. This is analogous to the Hall and Smith (1988) oblique-wave/vortex analysis in the TS case, and includes secondary 3D instability at the start, as does the full system of Section 4.

(iii) Wide-vortex/wave interactions similar to those in Section 2 can arise as limit cases of (4.2) - (4.4) for enlarged spanwise scales.

(iv) Special ranges of the Mach number $M_{\infty}$ are of theoretical and practical interest, including zero $M_{\infty}$ (see also Appendix B), small $M_{\infty}$, the transonic range $M_{\infty} \rightarrow 1$, and the hypersonic range of large $M_{\infty}$. These have connections, in turn, with the vast literature on linear incompressible Rayleigh modes in boundary layers, with Gajjar's (1989) linear and nonlinear critical-layer work, with Bowles' (1989) linear and nonlinear instability work, and with recent studies of linear hypersonic-flow instabilities. Concerning the hypersonic range in particular, the undisturbed steady $2 \mathrm{D}$ boundary layer with no imposed pressure gradient itself acquires a two-layered form at large Mach numbers (Bush (1966), Lee and Cheng (1969), Stewartson (1964)), with a relatively wide high-temperature layer, wherein $\bar{y}$ is $0\left(M_{\infty}^{2}\right)$, at the upper edge of which is a relatively thin high-vorticity layer with $\bar{y}-M_{\infty}^{2} F(x)$ of order $\left(\ln M_{\infty}^{2}\right)^{\frac{1}{2}}$. Here $\bar{y}=M_{\infty}^{2} F(x)$ denotes the scaled boundary-layer displacement. In line with this, the linear instability modes split into two types, the so-called vorticity mode concentrated within the high vorticity layer and having the maximum growth rate (Brown and Smith (1989)), and the so-called acoustic modes which spread normally across the $O\left(M_{\infty}^{2}\right)$ layer and have smaller growth rates (Hall and Cowley (1989), Brown and Smith (1989)). These linear features agree quite well with Mack's results at large $M_{\infty}$ and suggest the two most likely structures of nonlinear vortex/compressible-Rayleigh interaction at large $M_{\infty}$, as follows. 
First, the high-vorticity form of nonlinear interaction occurs where

$$
\bar{y}=M_{\infty}^{2} F(x)+(2 \Gamma)^{-1} \hat{y}\left[\text { with } \Gamma \exp \left(\Gamma^{2}\right)=M_{\infty}^{2}\right]
$$

and the scalings operating are of the form

$$
\begin{aligned}
\bar{u}=1-\frac{\bar{u}_{1}}{M_{\infty}^{2}}, \bar{v} & =M_{\infty}^{2} F_{x} \bar{u}+2 \Gamma \bar{v}, \bar{w}=\frac{M_{\infty}}{(2 \Gamma)^{\frac{1}{2}}} \bar{W} \\
\bar{z} & =M_{\infty}(2 \Gamma)^{-\frac{b}{2}} \hat{z} \\
\bar{\rho} & \sim 1, \bar{T} \sim 1, \bar{\mu} \sim 1 .
\end{aligned}
$$

These scalings are implied by the Prandtl shift in $\bar{y}$, in (5.1a), which introduces in effect a large Görtler number $M_{\infty}^{2} F_{x x}$ (for the high-vorticity-layer flow) that is negative for flatsurface flow where $F \propto+x^{\frac{1}{2}}$. Thus the vortex becomes relatively "wide." It is also quasi-2D in the cross-flow plane, since its governing equations become

$$
\begin{gathered}
(\bar{\rho} \bar{V})_{\hat{y}}+(\bar{\rho} \bar{W})_{z}=0 \\
\bar{\rho} F_{x x}=-\hat{\pi}_{\hat{y}} \\
\bar{\rho}\left(\bar{V} \bar{W}_{\hat{\vartheta}}+\bar{W} \bar{W}_{\hat{z}}\right)=-\hat{\pi}_{\bar{z}}+\left(\bar{\mu} \bar{W}_{\hat{\vartheta}}\right)_{\hat{y}} \\
\bar{\rho}\left(\bar{V} \bar{T}_{\hat{\jmath}}+\bar{W} \bar{T}_{z}\right)=\sigma^{-1}\left(\bar{\mu} \bar{T}_{\hat{\vartheta}}\right)_{\hat{\vartheta}} \\
\bar{\rho} \bar{T}=1, \bar{\mu}=C \bar{T} \\
\bar{\rho}\left(\bar{V} \bar{u}_{1 \hat{y}}+\bar{W} \bar{u}_{1 \hat{z}}\right)=\left(\overline{\mu u}_{1 \hat{y}}\right)_{\hat{\vartheta}}
\end{gathered}
$$

from (4.2) with (5.1). The coupled wave-amplitude equation is now

$$
\hat{p}_{\hat{y} \vartheta}-\frac{2 \hat{M}_{\hat{\vartheta}}}{\hat{M}} \hat{p}_{\hat{y}}-\hat{\alpha}^{2} \hat{p}=0
$$

where

$$
\left.\hat{M} \equiv\left(c_{1}-\bar{u}_{1}\right)\right)^{\frac{1}{2}},
$$

however, upon suitable scaling in (4.3), while the back reaction of the wave on the vortex motion is felt through the slip condition

$$
\bar{W}=-\frac{1}{3 C \gamma^{2}\left(\bar{u}_{1}^{\prime}\left(\hat{y}_{c}\right)\right)^{2}} \partial_{\hat{z}}\left(|\hat{p}|^{2}\right)
$$

at the unknown critical-level curve, from (4.4). Hence, despite the quasi-planar balances in (5.2a-f), the streamwise momentum balance $(5.2 \mathrm{~g})$ in the vortex still exerts influence on the nonlinear interaction due to the appearance of $\bar{u}_{1}$ in (5.3b) and (5.4). We observe that 
an alternative type of nonlinear interaction can arise either with sufficient surface curvature present, if the resulting Görtler number is equal to $M_{\infty}^{2} F_{x x}+0\left(\Gamma^{-1}\right)$, or with negligible curvature $F_{x x}$, since then the vortex can remain "square", more like those in Section 4 and below. Again, for small wave amplitudes linearized properties hold, with (5.2), (5.4) reproducing the basic boundary-layer solution via the Howarth-Dorodnitsyn transformation, while (5.3) then gives the equation addressed by Brown and Smith for which the neutral wavenumber is $\hat{\alpha}=\frac{1}{4}$. Comments similar to those in (i), (ii) above thereafter apply. There is also a neat, exact, local solution to the full system (5.2) - (5.4), described in Walton (1990), and a possible link with the experimental studies of Holden (1985), where pronounced rope-like vortex motions were observed at the edges of hypersonic boundary layers in the Mach-number range 11-13.

The second of the large- $M_{\infty}$ interaction structures is rather simpler, its scales being implied by the $0\left(M_{\infty}^{2}\right)$ thick boundary-layer form. Thus here

$$
\begin{gathered}
\left(\bar{y}, \bar{z}, \bar{T}, \bar{v}, \bar{w}, p_{2}\right) \sim M_{\infty}^{2} \\
\bar{u}=0(1),(\bar{\rho}, \alpha) \sim M_{\infty}^{-2}, \tilde{p} \sim M_{\infty}^{4}
\end{gathered}
$$

leaving the complete nonlinear interaction system (4.2) - (4.4) intact. The only new feature here is that the boundary-layer's normal extent becomes finite, $0 \leq \bar{y}<\bar{F}$ say, in normalized terms. The linearized version then matches up with the linear acoustic-modes analyses of Hall and Cowley and Brown and Smith, after which comments as in (i), (ii) again apply. It may be significant however that there are infinitely many acoustic modes, and hence possible bifurcations, available usually as the flow proceeds downstream, in contrast with the single vorticity mode; and the modes present with large wavenumbers, as well as mode-crossing, may shed extra light on the nonlinear interaction process.

(v) Extreme surface conditions, values of the parameters present such as $\sigma, C$, or pressure gradients, could also provide extra insight.

(vi) Besides the many applications within boundary-layer transition summarized above, and the corresponding computational tasks, and nonlinear similarity forms for instance, there are also many other flow configurations to which vortex/Rayleigh-wave interaction applies in principle (see Section 6 and Appendix B).

(vii) Finally here, we should mention the possibility that the Smith and Walton (1988, 1989) breakdown summarized in (3.2) also describes the ultimate behavior of the present vortex/compressible-Rayleigh nonlinear systems, at least for wide vortices. If so, the boundary layer again separates, effectively, splitting into two increasingly far-apart layers, with only slow motion in-between, at a finite distance downstream. That opens up an intriguing prospect, namely that a vortex/wave interaction (either as in Section 2 or as in Section 4) 
can induce separation (as above), which then introduces an extra (inflexional) inviscid mode and thence an extra vortex/wave nonlinear interaction, which leads to another separation, hence another interaction, and so on. This self-generating process causes the whole flow structure locally to cascade into smaller length scales, and thence into substructures.

\section{FURTHER COMMENTS}

The present work extends our previous studies of vortex/wave interaction (Hall and Smith (1987-1989)) to the strongly nonlinear regime in which the entire mean-flow profile, at any station $x$, is altered substantially from its undisturbed laminar form. This is for the compressible boundary-layer setting in the nonlinear vortex/Rayleigh interaction of Section 4 (the incompressible version is noted in Appendix B) and for the incompressible case in the nonlinear vortex/TS interaction of Section 2, with the corresponding compressible vortex/TS interaction following readily from a combination of those two sections (see also Smith and Walton $(1988,1989)$ ) and below. The solution properties and suggestions given in Section 3 , Section 5 hint fairly strongly, we believe, at the potential power of these nonlinear interactions in terms of full transition of the flow. In addition, it appears that numerous waves can be triggered off (see Section 5), all interacting nonlinearly with the unknown mean flow, especially if separation for instance is approached. These and other features of the nonlinear interactions found offer exciting prospects for more complete theoretical understanding of fully fledged boundary-layer transition.

Some numerical work for fully nonlinear interaction is described in Section 3, for the vortex/TS case, but further concerted efforts on full computations, for both the vortex/TS and the vortex/Rayleigh cases, are undoubtedly necessary and these represent a major challenge. They should enable quantitative comparisons with experiments and direct numerical simulations to be made eventually (an encouraging point being that qualitatively the flow structures in Section 2, Section 4 seem to be in line with the numerical-simulation experience, e.g., of Kleiser, that many more spanwise than streamwise wavenumbers are required to accurately describe fully fledged transition, except in its later stages). The pure spatial problem, the pure temporal problem, and the combined problem featuring the operator $\partial_{t}+\bar{u} \partial_{x}$ are all of interest here since they are likely to provoke different ultimate behaviors and hence possibly different views of such phenomena as lambda-vortex formations (see above) and the successive collapses in scales referred to in Section 5. The latter in turn may lead on to the 3D Euler stage locally, cf. Smith (1988) for nonlinear TS transition, allowing comparisons with experiments and direct numerical simulations (e.g., Zang, Erlebacher, Hussaini, Kleiser, and Biringen) on the later stages of transition, including spikes, intermittency and streak production. In all this, however, the computational tasks set by the nonlinear interaction 
problems posed in Section 2 and in (4.2) - (4.4) for instance seem to present a main hurdle.

Certain interesting other aspects of the vortex/wave interactions should also be put on record here. These are: various limiting or simplified cases, such as those in Section 5; similarity solutions (c.f., Section 3, Walton (1990)); the analogy with Görtler-vortex development (based on Hall $(1982,1983)$ and subsequent works); non-equilibrium critical-layer effects (in the context of Section 4); high-frequency properties (for Section 2, Section 4); and the implications for by-pass transition where the nonlinear interactions do not start from linear small-disturbance growth.

The nonlinear vortex/wave interactions also have numerous potential applications for other types of flows, in all of which the essential ideas of Section 2 and/or Section 4 would seem to apply: in wakes, channel flows (Bennett et al. (1988)), pipe flows (Walton, 1990), plane Couette flow, water motions, free shear layers, separations, vortex breakdown, where a swirl-velocity component is added to the vortex motion (and separation such as in (3.2) could correspond to abrupt vortex thickening), and flows over surface roughnesses, for example. See also Appendix B. The ideas apply further to length scales other than those taken in Section 2, Section 4. Again, there may be extensions of interest along the following lines: upperbranch flow structures, connected with the high-frequency limit; non-Chapman fluids and real-gas effects; surface-cooling effects; external-shock interactions in hypersonic flow, where the acoustic modes (Section 5) could play an important part; external pressure-gradient influences; and alternate compressible interactions. Nevertheless, the major challenge pressing seems to us to be the computational one of accurately solving the vortex/wave nonlinear interaction equations set up in Section 2, Section 4, given the encouraging guidelines on the strongly nonlinear effects possible. 


\section{References}

[1] Bennett, J., Hall, P., and Smith, F. T., 1988, ICASE Report No. 88-45, submitted to J. Fluid Mech.

[2] Blackaby, N. D., 1990, Ph.D. Thesis, Univ. of London.

[3] Bowles, R. I., 1989, Ph.D. Thesis, Univ. of London.

[4] Brown, S. N. and Smith, F. T., 1989, submitted to J. Fluid Mech.

[5] Bush, W. B., 1966, J. Fluid Mech. 25, 51.

[6] Cowley, S. J. and Hall, P., 1989, to appear in J. Fluid Mech.

[7] Fasel, H., Rist, U., and Konzelmann, U., 1987, AIAA Paper No. 87-1203.

[8] Gajjar, J. S. B., 1989, submitted to J. Fluid Mechanics.

[9] Gilbert, N. and Kleiser, L., 1986, in "Direct and Large Eddy Simulation of Turbulence," U. Schumann and R. Friedrich (Eds.), 1-18, Vieweg, Braunschweig.

[10] Gilbert, N. and Kleiser, L., 1988, Proc. Int. Seminar on Near-Wall Turbulence, Hemisphere, Washington, DC.

[11] Hama, F. R. and Nutant, J., 1963, Proc. Heat Transfer and Fluid Mechs. Inst., 77-93, Stanford University Press, Palo Alto, CA.

[12] Hall, P., 1982, J. Inst. Math. and its Applics. 29, 173-196.

[13] Hall, P., 1983, J. Fluid Mech. 130, 41-68.

[14] Hall, P. and Lakin, W. D., 1988, Proc. Roy. Soc. A 415, 421-444.

[15] Hall, P. and Smith, F. T., 1984, Studies in Appl. Math. 70, 91.

[16] Hall, P. and Smith, F. T., 1987, ICASE Report 87-25 and Proc. Roy. Soc. A 417, 255, 1988.

[17] Hall, P. and Smith, F. T., 1988, ICASE Report No. 88-46 and European J. Mech. B8, 179-205, 1989.

[18] Hall, P. and Smith, F. T., 1989, ICASE Report and to appear in Proc. ICASE Workshop on Instability and Transition. 
[19] Holden, M. S., 1985, AIAA Paper No. 85-0325, presented at Reno, NV, January 1985.

[20] Kachanov, Yu. S. and Levchenko, V. Yu, 1984, J. Fluid Mech. 138, 209-247.

[21] Klebanoff, P. S., Tidstrom, K. D., and Sargent, L. M., 1962, J. Fluid Mech. 12, 1-34.

[22] Kleiser, L. and Schumann, U., 1984, Proc. ICASE Symp. on Spectral Methods, R. G. Voigt, D. Gottlieb, and M. Y. Hussaini (Eds.), 141-163, SIAM-CBMS, Philadelphia, PA.

[23] Laurien, E. and Kleiser, L., 1989, J. Fluid Mech.

[24] Lee, R. S. and Cheng, H. K., 1969, J. Fluid Mech. 38, 161-179.

[25] Mack, L. M., 1975, AIAA J. 13, 278-289.

[26] Mack, L. M., 1984, AGARD Report 709.

[27] Malik, M. R., 1982, NASA CR-165925.

[28] Malik, M. R., 1987, AIAA Paper No. 87-1414.

[29] Nishioka, M., Asai, M., and Iida, S., 1981, in "Transition and Turbulence," R. E. Meyer (Ed.), 113-126, Academic Press, NY.

[30] Nishioka, M. and Asai, M., 1984, in "Turbulence and Chaotic Phen. in Fluids," T. Tatsumi (Ed.), 87-92, North-Holland, Amsterdam.

[31] Nishioka, M. and Asai, M., 1985, J. Fluid Mech. 150, 441-450.

[32] Smith, F. T., 1979, Proc. Roy. Soc. A 366, 91.

[33] Smith, F. T., 1985, UTRC Report 85-36 and J. Fluid Mech. 169, 353, 1986.

[34] Smith, F. T., 1987, UTRC Report 87-52 and J. Fluid Mech. 198, 127-153, 1989.

[35] Smith, F. T, 1988, Mathematika 35, 256-273.

[36] Smith, F. T., 1989, Computers and Fluids, to appear, Proceedings of R. T. Davis Memorial Symp., Cincinnati, 1987.

[37] Smith, F. T. and Burggraf, O. R., 1985, Proc. Roy. Soc. A 399, 25.

[38] Smith, F. T., Doorly, D. J., and Rothmayer, A. P., 1989, to appear in Proc. Roy. Soc. A; based on UTRC Report 87-43. 
[39] Smith, F. T. and Stewart, P. A., 1987, J. Fluid Mech. 179, 227-252.

[40] Smith, F. T. and Walton, A. G., 1988/1989, ICASE Report 88-66 and Mathematika 36, Part 2.

[41] Spalart, P. R. and Yang, K. S., 1987, J. Fluid Mech. 178, 345-365.

[42] Stewartson, K., 1964, "The theory of laminar boundary layers in compressible fluids," Oxford Univ. Press.

[43] Thomas, A. S. W., 1987, Proc. 10th U. S. Natl. Congress on Applied Math., 436-444, ASME, NY.

[44] Walton, A. G., 1990, Ph.D. Thesis, University of London.

[45] Williams, D. R., Fasel, H., and Hama, F. R., 1984, J. Fluid Mech. 149, 179-203.

[46] Williams, D. R., 1987, in "Stability of time dependent and spatially varying flows," D. L. Dwoyer and M. Y. Hussaini (Eds.), 335-350, Springer-Verlag, NY.

[47] Wray, A. A. and Hussaini, M. Y., 1984, Proc. Roy. Soc. A 392, 373-389.

[48] Zang, T. A. and Hussaini, M. Y., 1986, Applied Math. Comput. 19, 359-372.

[49] Zang, T. A. and Hussaini, M. Y., 1987, in "Nonlinear wave interactions in fluids," R. W. Miksad, T. R. Akylas, T. Herbert (Eds.), 131-145, AMD 87, ASME, NY.

[50] Zang, T. A. and Krist, S. E., 1989, Theor. Comp. Fluid Dyn. 1, 41-64. 


\section{APPENDIX A. THE CRITICAL-LAYER BEHAVIOR}

The critical layer occurs when $\bar{y}=f(x, \bar{z})+\operatorname{Re}^{-\frac{1}{6}} Y$, with $Y$ of $0(1)$, and the flow properties produced are predominantly linear. The solution takes the form

$$
\begin{gathered}
u=c+\operatorname{Re}^{-\frac{1}{6}} \lambda_{1}(x, \bar{z}) Y+\ldots+\operatorname{Re}^{-\frac{1}{3}} \mathcal{L}_{1} U^{(1)}+\ldots+\operatorname{Re}^{-\frac{1}{3}}\left(\mathcal{L}_{1}^{2} \bar{U}+\mathcal{L}_{1} U^{(2)}\right)+\ldots, \\
v=\ldots+\operatorname{Re}^{-\frac{1}{3}} \mathcal{L}_{1} V^{(1)}+\operatorname{Re}^{-\frac{1}{2}}\left(\mathcal{L}_{1}^{2} f_{\bar{z}} \bar{W}+\mathcal{L}_{1} V^{(2)}\right)+\ldots+\operatorname{Re}^{-\frac{2}{3}} \mathcal{L}_{1}^{2} \bar{V}+\ldots, \\
w=\ldots+\operatorname{Re}^{-\frac{1}{3}} \mathcal{L}_{1} W^{(1)}+\ldots+\operatorname{Re}^{-\frac{1}{2}}\left(\mathcal{L}_{1}^{2} \bar{W}+\mathcal{L}_{1} W^{(2)}\right)+\ldots \\
p=\ldots+\operatorname{Re}^{-\frac{1}{2}} \mathcal{L}_{1} P^{(1)}+\operatorname{Re}^{-\frac{2}{3}} \mathcal{L}_{1} P^{(2)}+\ldots
\end{gathered}
$$

where $\lambda_{1}$ is the streamwise vortex shear at the critical layer, the main wave part (superscript (1)) depends on the fast scales $(X, \bar{t})=\operatorname{Re}^{\frac{1}{2}}(x, t)$ with wavespeed $c$ as in Section 4, and the main vortex part (overbarred) is independent of the fast scales. Here we discuss the incompressible case with zero pressure gradient, for the sake of clarity; the compressible version follows along similar lines.

The successive balances in mass conservation resulting from (A1) - (A4) give

$$
\begin{gathered}
V_{Y}^{(1)}=f_{\bar{z}} W_{Y}^{(1)}, \\
U_{X}^{(1)}+V_{Y}^{(2)}+W_{\bar{z}}^{(1)}-f_{\bar{z}} W_{Y}^{(2)}=0 \\
-f_{X} \lambda_{1}+\bar{V}_{Y}+W_{\bar{z}}=0
\end{gathered}
$$

and the $x$-momentum balances of concern are

$$
\begin{gathered}
V^{(1)}=f_{\bar{z}} W^{(1)}, \\
\lambda_{1} Y U_{X}^{(1)}+\lambda_{1} V^{(2)}+W^{(1)} \lambda_{1 \bar{z}} Y-W^{(2)} f_{\bar{z}} \lambda_{1}=-P_{X}^{(1)}+\Delta U_{Y Y}^{(1)}, \\
<U^{(1)} U_{X}^{(1)}+V^{(2)} U_{Y}^{(1)}+W^{(1)} U_{\bar{z}}^{(1)}-W^{(2)} f_{\bar{z}} U_{Y}^{(1)}>+\bar{V} \lambda_{1}+\bar{W} Y \lambda_{1 \bar{z}}=\ldots+\Delta \bar{U}_{Y Y} .
\end{gathered}
$$

Likewise, the $z$-momentum balances here become

$$
\begin{gathered}
\lambda_{1} Y W_{X}^{(1)}=-P_{\bar{z}}^{(1)}+f_{\bar{z}} P_{Y}^{(2)}+\Delta W_{Y Y}^{(1)} \\
<U^{(1)} W_{X}^{(1)}+V^{(2)} W_{Y}^{(1)}+W^{(1)} W_{\bar{z}}^{(1)}-W^{(2)} f_{\bar{z}} W_{Y}^{(1)}>=\ldots+\Delta \bar{W}_{Y Y},
\end{gathered}
$$

while the $y$-momentum equation implies that $P^{(1)}$ is independent of $Y$, and hence equal to $p^{(1)}$, and

$$
P_{Y}^{(2)}=-\lambda_{1} Y V_{X}^{(1)}+\Delta V_{Y Y}^{(1)}
$$

In (A10), (A12), <> refers to the vortex components only, in the enclosed terms. 
The wave part in this non-flat critical layer may be analyzed by putting $V^{(2)}-f_{\bar{z}} W^{(2)}=$ $V_{a}$, say, which leaves $U^{(1)}, V_{a}, W^{(1)}$ satisfying essentially the equations of a linear 3D disturbance in a standard flat critical layer, from (A6), (A9), (A11), (A13) and since $V^{(1)}=f_{\bar{z}} W^{(1)}$ from (A5), (A8). In particular, $W^{(1)}$ satisfies a forced Airy equation in the form

$$
\lambda_{1} Y W_{X}^{(1)}=-P_{\bar{z}}^{(1)} \Delta^{-1}+\Delta W_{Y Y}^{(1)}
$$

which yields the solution

$$
W^{(1)}=-\left(\Delta^{2} \lambda_{1} i \alpha\right)^{-\frac{2}{3}} \tilde{p}_{\bar{z}} \mathcal{J}(\hat{Y}) E+c . c ., \quad Y=\left(\frac{\Delta}{\lambda_{1} i \alpha}\right)^{\frac{1}{3}} \hat{Y}
$$

where $\mathcal{J}$ satisfies $\mathcal{J}^{\prime \prime}-\hat{Y} \mathcal{J}=1, \mathcal{J}( \pm \infty)=0$, and can be expressed in terms of the Airy function, and $E=\exp (i \alpha X-i \Omega \tilde{t})$. This and the corresponding solutions for $V_{a}, U^{(1)}$ are smooth for all $Y$ and satisfy the asymptotic conditions of matching,

$\left[U^{(1)}, V^{(1)}, W^{(1)}\right] \sim Y^{-1}\left[F_{-1}, G_{-1}, H_{-1}\right] E+c . c .,\left[V^{(2)}, W^{(2)}\right] \rightarrow\left[G_{0}, H_{0}\right] E+c . c ., \quad$ as $Y \rightarrow \pm \infty$,

implied by (4.7), (4.8) (with Appendix B), as required.

Then the main vortex equation of interest here is that for $\bar{W}$, in (A12). Here the behaviour in (A16) shows that the nonlinear forcing term on the left-hand side of (A12) decays as $Y^{\mathbf{- 2}}$ as $Y \rightarrow \pm \infty$. So double integration of (A12) with respect to $Y$ produces the response

$$
\bar{W} \sim-\hat{B} \ell n|Y| \text { as } Y \rightarrow \pm \infty
$$

with $\hat{B}=2\left(H_{-1} H_{-1}^{*}\right)_{\bar{z}}+\left(f_{\bar{z}} H_{0}-G_{0}\right) H_{-1}^{*}+\left(f_{\bar{z}} H_{0}^{*}-G_{0}^{*}\right) H_{-1}$ being found to equal $-\bar{q} \Delta^{-\frac{1}{2}}$ as defined in (4.4b) or in Appendix B. The logarithmic behavior in (A17) and the associated logarithmic terms in $\bar{U}, \bar{V}$ at large $|Y|$ are responsible for the scale factor $\mathcal{L}_{1}$ in (4.1), as well as for the effective slip condition in (4.4b). The condition (4.4b) follows from the match with the flow solution in Section 4, since $\ell n|Y| \rightarrow \frac{1}{6} \ln \operatorname{Re}+\ln |\bar{y}-f|$ in effect. 


\section{APPENDIX B. VORTEX/RAYLEIGH-WAVE INTERACTIONS IN THE IN- COMPRESSIBLE CASE}

Many of the extra applications mentioned in the text are concerned more with incompressible fluids, for which the vortex/wave interaction equations of (4.2) - (4.4) continue to hold provided $\bar{\rho}, \bar{\mu}$ are replaced by unity in (4.2a-d), $\bar{M}$ is replaced by $(\bar{u}-c)$ in (4.3a), with the $\bar{M}^{2}$ term omitted, and (4.4b) is replaced by

$$
\bar{q}=-\frac{\Delta^{-\frac{s}{2}}}{\left(\bar{u}_{1}\right)^{2} \alpha^{2}}\{\}
$$

where the curly brackets signify the curly-bracketed expression in (4.4b). The governing equations in that case are $(4.2 \mathrm{a}-\mathrm{d}, \mathrm{h}, \mathrm{i}),(4.3 \mathrm{a}-\mathrm{c}),(4.4 \mathrm{~b}-\mathrm{d})$, with the above modifications. In addition, the local pressure expansion (4.5a) remains valid provided that in (4.6a-c) $\bar{M}_{1}, \bar{M}_{2}$ are replaced by $\bar{u}_{1}, \bar{u}_{2}$ respectively. Similarly, the local velocity expansions (4.7a-c) remain true provided that

$$
G_{-1}=-\tilde{p}_{1} / i \alpha \bar{u}_{1}, H_{-1}=\left(f_{\bar{z}} \tilde{p}_{1}-\tilde{p}_{0 \bar{z}}\right) / i \alpha \bar{u}_{1}, F_{-1}=-H_{-1 \bar{z}} / i \alpha
$$

instead of (4.8a-c) in turn; also

$$
\begin{gathered}
G_{0}=-\bar{u}_{2} G_{-1} / \bar{u}_{1}-2 \tilde{p}_{2} / i \alpha \bar{u}_{1}, \\
H_{0}=-\bar{u}_{2} H_{-1} / \bar{u}_{1}+\left(2 f_{\bar{z}} \tilde{p}_{2}-\tilde{p}_{1 \bar{z}}\right) / i \alpha \bar{u}_{1}
\end{gathered}
$$

in this case. 

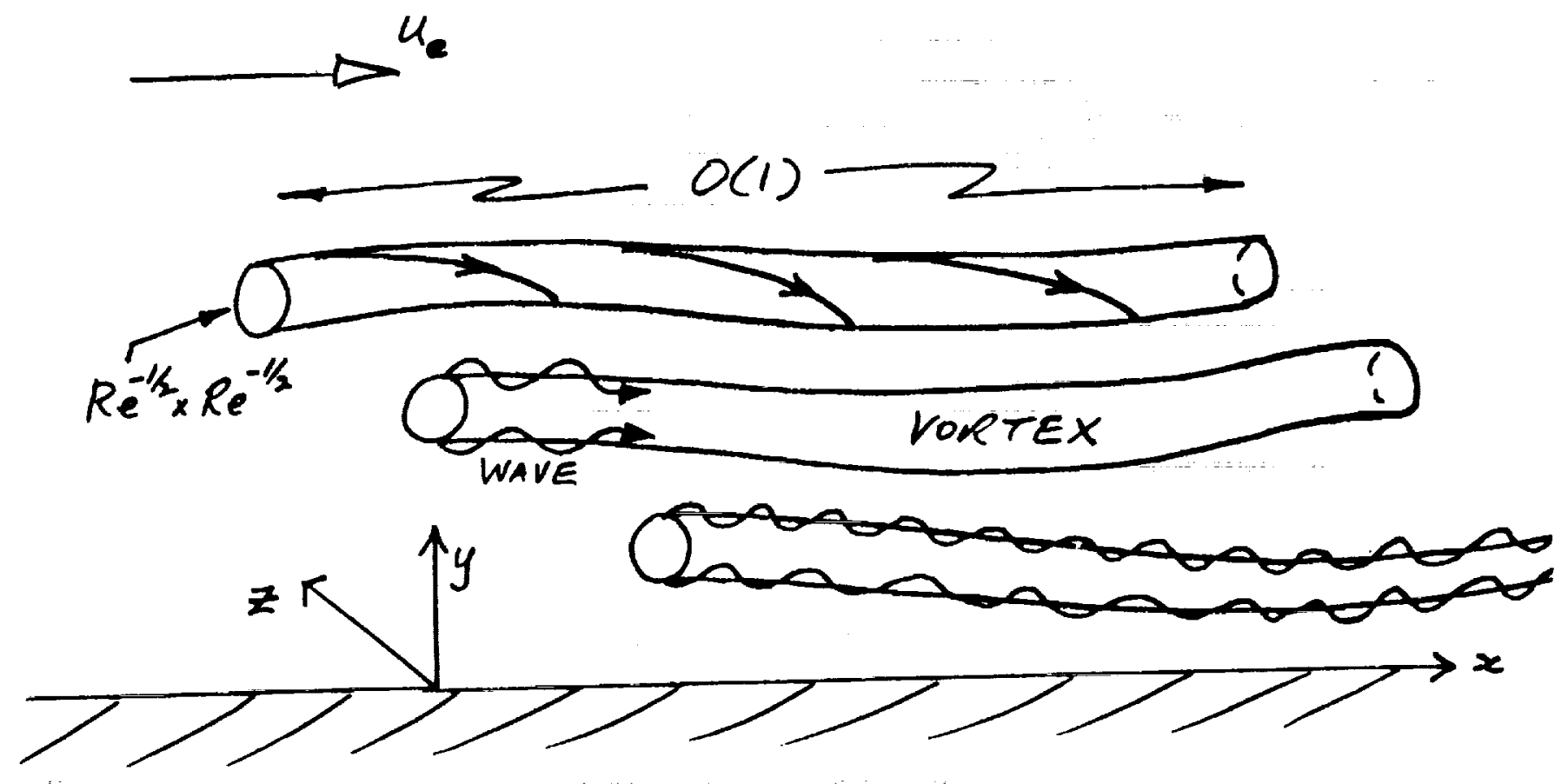

Figure 1 (a) Typical strongly nonlinear vortex/wave interactions in a boundary layer. The scales of the slowly varying vortices are shown; the travelling waves present are either TS waves (Sections 2, 3) or Rayleigh waves (Sections 4, 5). 


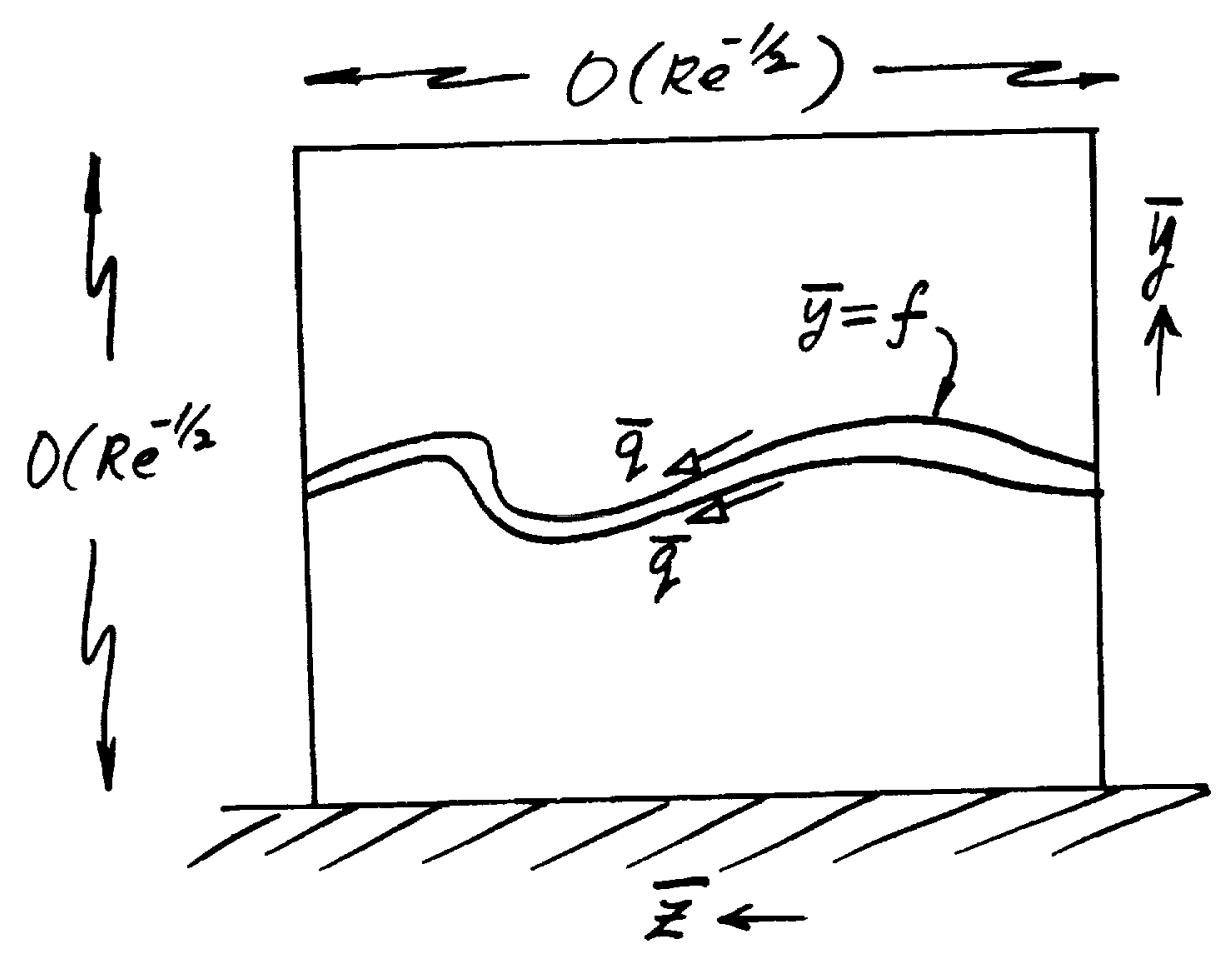

Figure 1 (b) Cross-section $O\left(\operatorname{Re}^{-\frac{1}{2}} \times \operatorname{Re}^{-\frac{1}{2}}\right)$ in the vortex/Rayleigh-wave case of Sections 4,5 , including the critical-layer $O\left(\operatorname{Re}^{-\frac{2}{3}}\right)$ thick) effect at $\bar{y}=f$. 


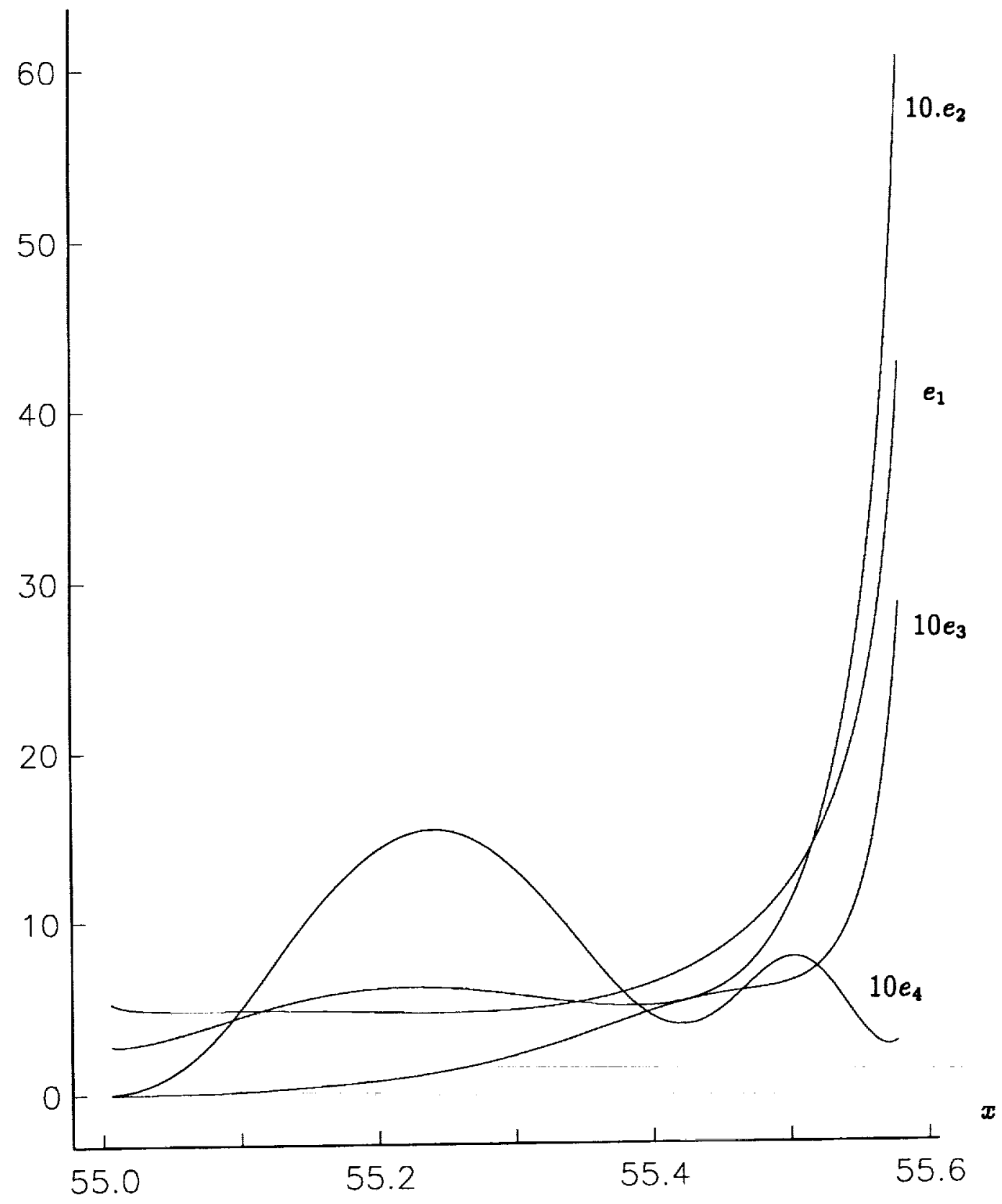

Figure 2(a) The development of $e_{1}, e_{2}, e_{3}, e_{4}$ with $n$ for the case $\Delta=.02, P_{0}+\sum_{1}^{\text {infty }} P_{n}=1$ at $x=55$. 


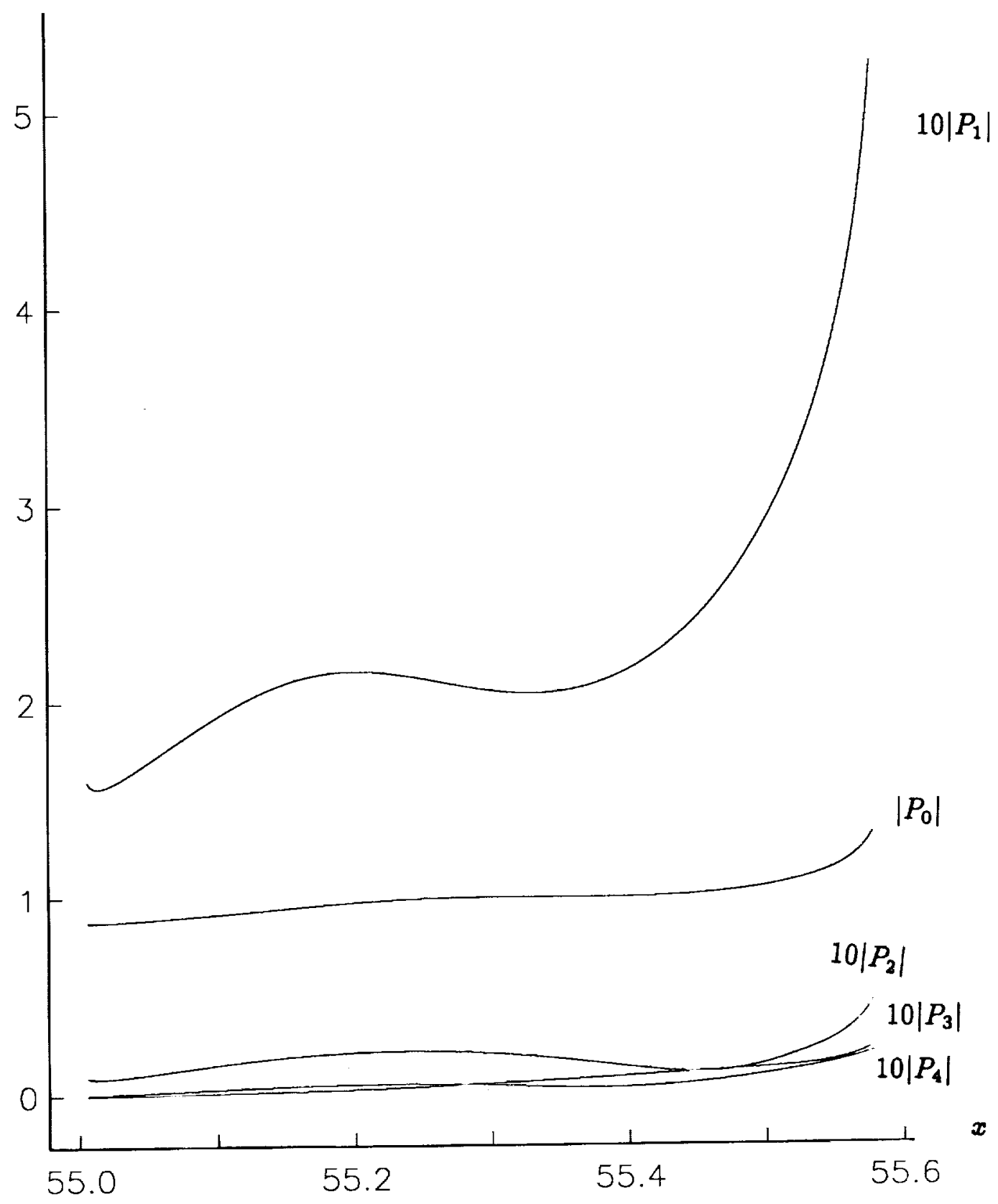

Figure 2(b) The development of $P_{0}, P_{1}, P_{2}, P_{3}, P_{4}$ with $x$ for the case $\Delta=.02$, $P_{0}+\Sigma_{1}^{\text {infty }} P_{n}=1$ at $x=55$. 


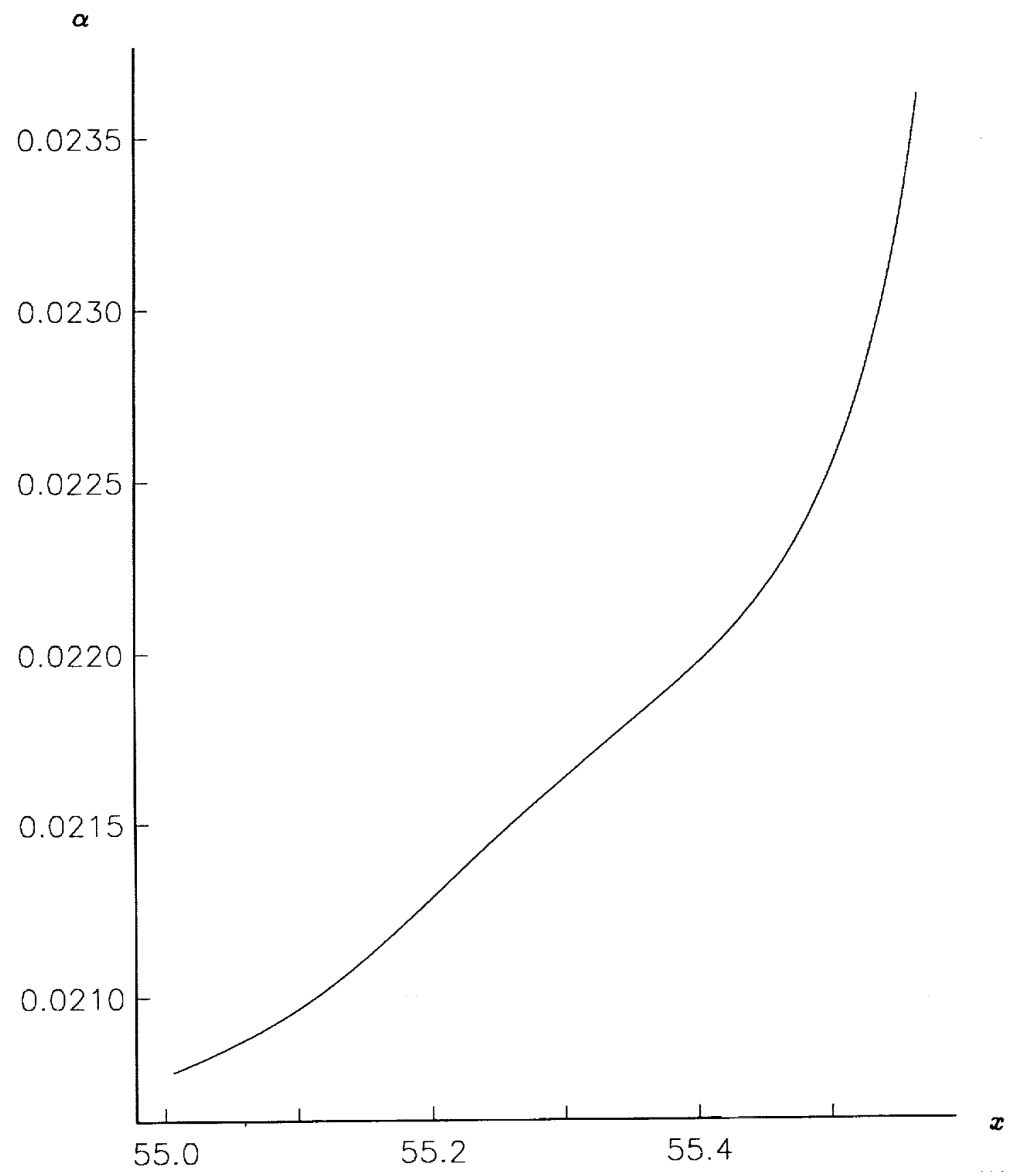

Figure 2(c) The development of $\alpha$ with $n$ for the case $\Delta=.02, P_{0}+\sum_{1}^{i n f t y} P_{n}=1$ at $x=55$. 


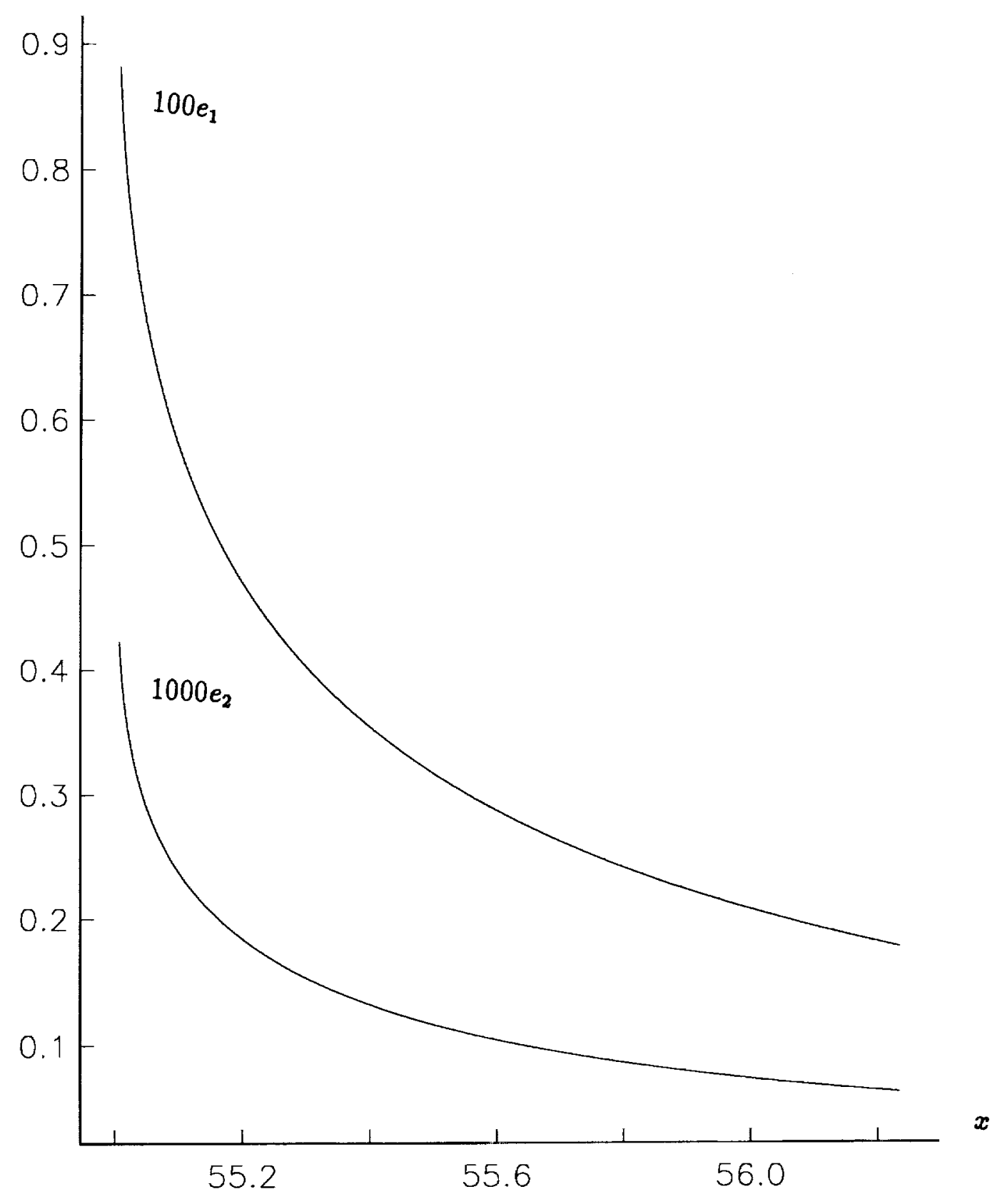

Figure 3(a) The development of $e_{1}, e_{2}$ with $n$ for the case $\Delta=.02, P_{0}+\sum_{1}^{\text {infty }} P_{n}=.2$ at $x=55$. 


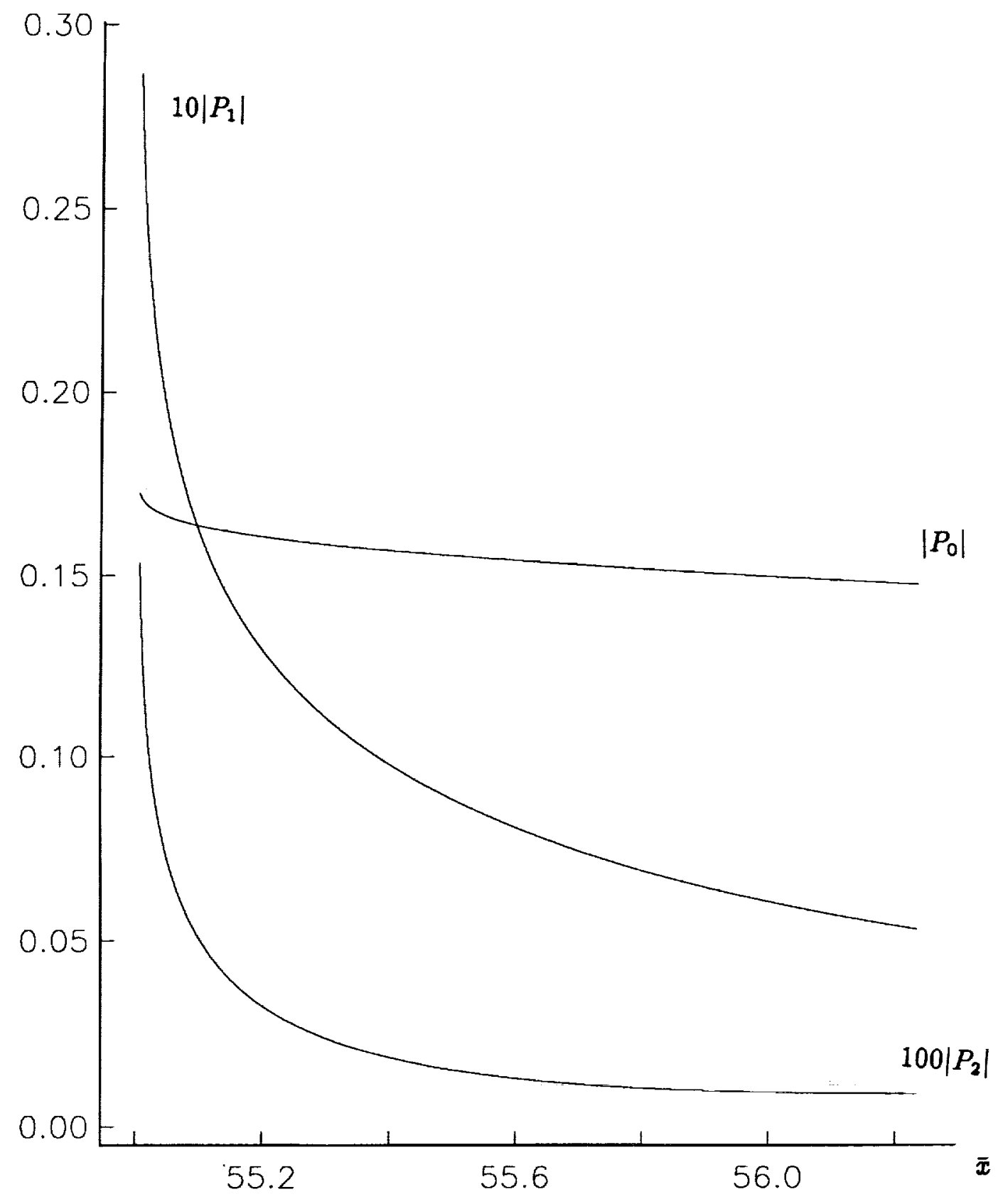

Figure $3(\mathrm{~b})$ The development of $P_{0}, P_{1}, P_{2}$ with $x$ for the case $\Delta=.02, P_{0}+\sum_{1}^{\text {infty }} P_{n}=.2$ at $\bar{x}=55$. 

. 


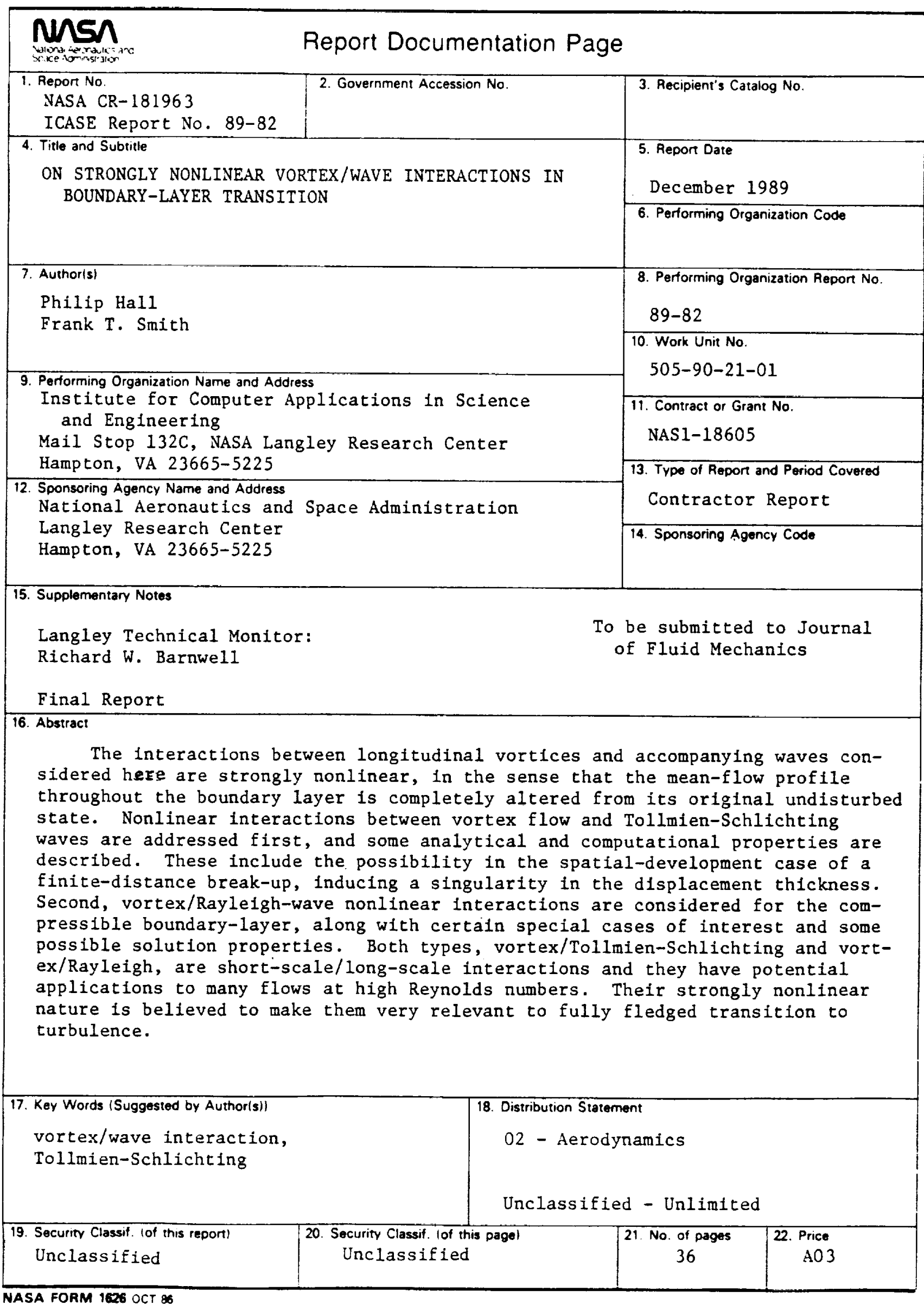


